\title{
Analysis and Numerical Simulations of a Stochastic SEIQR Epidemic System with Quarantine-Adjusted Incidence and Imperfect Vaccination
}

\author{
Fei Li, ${ }^{1}$ Xinzhu Meng $\left(\mathbb{D},{ }^{1,2}\right.$ and Xinzeng Wang ${ }^{1,2}$ \\ ${ }^{1}$ College of Mathematics and Systems Science, Shandong University of Science and Technology, Qingdao 266590, China \\ ${ }^{2}$ State Key Laboratory of Mining Disaster Prevention and Control Co-Founded by Shandong Province and the Ministry of \\ Science and Technology, Shandong University of Science and Technology, Qingdao 266590, China
}

Correspondence should be addressed to Xinzhu Meng; mxz721106@sdust.edu.cn

Received 21 October 2017; Accepted 28 January 2018; Published 20 February 2018

Academic Editor: Xiaole Chen

Copyright (C) 2018 Fei Li et al. This is an open access article distributed under the Creative Commons Attribution License, which permits unrestricted use, distribution, and reproduction in any medium, provided the original work is properly cited.

\begin{abstract}
This paper considers a high-dimensional stochastic SEIQR (susceptible-exposed-infected-quarantined-recovered) epidemic model with quarantine-adjusted incidence and the imperfect vaccination. The main aim of this study is to investigate stochastic effects on the SEIQR epidemic model and obtain its thresholds. We first obtain the sufficient condition for extinction of the disease of the stochastic system. Then, by using the theory of Hasminskii and the Lyapunov analysis methods, we show there is a unique stationary distribution of the stochastic system and it has an ergodic property, which means the infectious disease is prevalent. This implies that the stochastic disturbance is conducive to epidemic diseases control. At last, computer numerical simulations are carried out to illustrate our theoretical results.
\end{abstract}

\section{Introduction}

Mathematical models for differential equations have been widely applied in various fields [1-7]. Specifically, they have had a realistic significance to analyze the dynamical behaviors in the field of mathematical biology [8-17], which obtained some novel results.

In fact, the main meaning of the research of infectious disease dynamics is to make people more comprehensively and deeply understand the epidemic regularity of infectious disease; then more effective control strategies are adopted to provide better theoretical support for the prevention and control of epidemics. To this end, many mathematical biology workers considered more realistic factors in the course of the study, such as population size change, migration, cross infection, and other practical factors. In the course of epidemics and outbreaks of infectious diseases, people always take various measures to control the epidemic in order to minimize the harm of epidemic diseases. Quarantine is one of the important means to prevent and control epidemic diseases; it has been used to control contagious diseases with some success. Specifically, during the severe acute respiratory syndrome (SARS) outbreak in 2002, remarkable results were also achieved. Among them, mathematical models have been used to investigate their impact on the dynamics of infectious diseases under quarantine [18-22], which attracts deep research interest of many mathematicians and biologists. Recently, Hethcote et al. [21] considered an SIQR (susceptible-infected-quarantined-recovered) model with quarantine-adjusted incidence. The system can be expressed as follows:

$$
\begin{aligned}
\dot{S} & =\Lambda-\frac{\beta S I}{\widetilde{N}-Q}-\mu S, \\
\dot{I} & =\frac{\beta S I}{\widetilde{N}-Q}-\left(\lambda+\varepsilon_{1}+\gamma+\mu\right) I, \\
\dot{Q} & =\lambda I-\left(\varphi+\varepsilon_{2}+\mu\right) Q, \\
\dot{R} & =\gamma I+\varphi Q-\mu R,
\end{aligned}
$$

where the total population size is given by $\widetilde{N}(t)=S(t)+$ $I(t)+Q(t)+R(t), \Lambda$ is the inflow rate corresponding to birth 
and immigration, and $\mu$ is the outflow rate corresponding to natural death and emigration. Since the quarantine process, using the standard incidence $(\beta I / \widetilde{N}) S$, the contact rate $\beta Q / \widetilde{N}$ with the quarantined fraction $Q / \widetilde{N}$ does not occur. Hence the standard incidence is replaced by $\beta S I /(\widetilde{N}-Q)$ (it is called quarantine-adjusted incidence); here $\beta$ is the transmission coefficient between susceptible individuals and infected individuals. $\lambda$ is the quarantine rate of infected individuals, $\varphi$ is the recovery rate of quarantined individuals, and $\varepsilon_{1}$ and $\varepsilon_{2}$ stand for the rate of disease-related death of infected and quarantined individuals, respectively. $\gamma$ is the recovery rate of infected individuals. Furthermore, all the parameters are positive and the region $\widetilde{D}=\{(S, I, Q, R) \mid S \geq 0, I \geq$ $0, Q \geq 0, R \geq 0, S+I+Q+R \leq \Lambda / \mu\}$ is a positively invariant set of system (1). In the region $\widetilde{D}$, they established the basic reproduction number $R_{0}$, which determines disease extinction or permanence, where

$$
R_{0}=\frac{\beta}{\lambda+\varepsilon_{1}+\gamma+\mu} .
$$

Meanwhile, they analyzed the global dynamics of system (1) and derived the equilibria (including the disease-free equilibrium and the endemic equilibrium) and their global stability. In addition, the parameter conditions for the existence of a Hopf bifurcation are obtained.

In the real world, with the development of modern medicine, vaccination has become an important strategy for disease prevention and control in addition to quarantine, and numerous scholars have investigated the effect of vaccination on disease [23-30]. For another, many infectious diseases incubate inside the hosts for a period of time before becoming infectious, so it is very meaningful to consider the effect of the incubation period. Motivated by the aforementioned work, this paper considers an SEIQR (susceptibleexposed-infected-quarantined-recovered) epidemic model with imperfect vaccination, which is described by the following system:

$$
\begin{aligned}
& \dot{S}=\Lambda-\frac{(1-\delta) \beta S I}{N-Q}-\frac{\delta(1-p) \beta S I}{N-Q}-(\delta p+\mu) S, \\
& \dot{E}=\frac{(1-\delta) \beta S I}{N-Q}+\frac{\delta(1-p) \beta S I}{N-Q}-(\alpha+\mu) E, \\
& \dot{I}=\alpha E-\left(\lambda+\varepsilon_{1}+\gamma+\mu\right) I, \\
& \dot{Q}=\lambda I-\left(\varphi+\varepsilon_{2}+\mu\right) Q \\
& \dot{R}=\delta p S+\gamma I+\varphi Q-\mu R,
\end{aligned}
$$

where the total population size is given by $N(t)=S(t)+E(t)+$ $I(t)+Q(t)+R(t), \delta(0 \leq \delta<1)$ is the vaccine coverage rate, $p(0 \leq p \leq 1)$ is the vaccine efficacy, and $\alpha$ is the rate at which the exposed individuals become infected individuals. Other parameters are the same as in system (1). Now we assume that all the parameters are positive constants here except that $\delta, p$ are nonnegative constants. Clearly, the region $D=\{(S, E, I, Q, R) \mid S \geq 0, E \geq 0, I \geq 0, Q \geq 0, R \geq 0$,
$S+E+I+Q+R \leq \Lambda / \mu\}$ is a positively invariant set of system (3). For system (3), the basic reproduction number is

$$
R_{1}=\frac{\mu(1-\delta p) \beta \alpha}{(\delta p+\mu)(\alpha+\mu)\left(\lambda+\varepsilon_{1}+\gamma+\mu\right)}
$$

and it has the following properties:

(1) When $R_{1} \leq 1$ holds, system (3) has a unique diseasefree equilibrium $E_{0}=\left(S_{0}, 0,0,0, R_{0}\right)=(\Lambda /(\delta p+$ $\mu), 0,0,0, \delta p \Lambda / \mu(\delta p+\mu))$ which is globally asymptotically stable in the region $D$. That means the epidemic diseases will die out and the total individuals will become the susceptible and recovered individuals.

(2) When $R_{1}>1$ holds, system (3) has a unique globally asymptotically stable positive equilibrium $E^{*}=\left(S^{*}, E^{*}, I^{*}, Q^{*}, R^{*}\right)$ in the region $D$, which means the epidemic diseases will persist.

In the natural world, deterministic model is not enough to describe the species activities. Sometimes, the species activities may be disturbed by uncertain environmental noises. Consequently, some parameters should be stochastic [31-40]. There is no denying that this phenomenon is ubiquitous in the ecosystem. Therefore numerous scholars have introduced the effect of stochastic perturbation on diseases [41-50]. To the best of our knowledge, the research on global dynamics of the stochastic SEIQR epidemic model with imperfect vaccination is not too much yet. In this paper, to make system (3) more reasonable and realistic, we assume the environmental noise is directly proportional to $S(t), E(t), I(t), Q(t)$, and $R(t)$. Then corresponding to system (3), a stochastic version can be reached by

$\mathrm{d} S$

$$
\begin{aligned}
= & {\left[\Lambda-\frac{(1-\delta) \beta S I}{N-Q}-\frac{\delta(1-p) \beta S I}{N-Q}-(\delta p+\mu) S\right] \mathrm{d} t } \\
& +\sigma_{1} S \mathrm{~d} B_{1}(t),
\end{aligned}
$$

$\mathrm{d} E$

$$
\begin{aligned}
= & {\left[\frac{(1-\delta) \beta S I}{N-Q}+\frac{\delta(1-p) \beta S I}{N-Q}-(\alpha+\mu) E\right] \mathrm{d} t } \\
& +\sigma_{2} E \mathrm{~d} B_{2}(t), \\
\mathrm{d} I= & {\left[\alpha E-\left(\lambda+\varepsilon_{1}+\gamma+\mu\right) I\right] \mathrm{d} t+\sigma_{3} I \mathrm{~d} B_{3}(t), } \\
\mathrm{d} Q= & {\left[\lambda I-\left(\varphi+\varepsilon_{2}+\mu\right) Q\right] \mathrm{d} t+\sigma_{4} Q \mathrm{~d} B_{4}(t), } \\
\mathrm{d} R= & (\delta p S+\gamma I+\varphi Q-\mu R) \mathrm{d} t+\sigma_{5} R \mathrm{~d} B_{5}(t),
\end{aligned}
$$

where $B_{i}(t)(i=1,2,3,4,5)$ is the mutually independent standard Wiener process with $B_{i}(0)=0$ a.s. $\sigma_{i}(t)(i=$ $1,2,3,4,5)$ is a continuous and bounded function for any $t \geq 0$ and $\sigma_{i}^{2}(t)(i=1,2,3,4,5)$ are the intensities of Wiener processes. 
In this paper, we are mainly concerned with two interesting problems as follows:

(P1) Under what parameter conditions, will the disease die out?

(P2) Under what conditions, will system (5) have a unique ergodic stationary distribution?

Throughout this paper, let $\left(\Omega, \mathscr{F},\{\mathscr{F}\}_{t \geq 0}, \mathbb{P}\right)$ be a complete probability space with a filtration $\left\{\mathscr{F}_{t}\right\}_{t \geq 0}$ satisfying the usual conditions (i.e., it is increasing and right continuous while $\mathscr{F}_{0}$ contains all $\mathbb{P}$-null sets $)$. Further $B_{i}(t)(i=$ $1,2,3,4,5)$ is defined on the complete probability space.

For simplicity and convenience, we introduce the following notations:

(1) $\mathbb{R}_{+}=[0,+\infty), \mathbb{R}_{+}^{5}=\left\{x=\left(x_{1}, x_{2}, x_{3}, x_{4}, x_{5}\right) \in \mathbb{R}^{5}\right.$ : $\left.x_{i}>0(i=1,2,3,4,5)\right\}$.

(2) For an integrable function $x(t) \in[0,+\infty),\langle x(t)\rangle=$ $(1 / t) \int_{0}^{t} x(r) \mathrm{d} r$

(3) $a \wedge b=\min \{a, b\}, a \vee b=\max \{a, b\}$.

\section{Global Positive Solution}

To investigate the dynamical behaviors of a population system, we first concern the global existence and positivity of the solutions of system (5).

Lemma 1. For any given initial value $(S(0), E(0), I(0), Q(0)$, $R(0)) \in \mathbb{R}_{+}^{5}$, system (5) has a unique positive local solution $(S(t), E(t), I(t), Q(t), R(t))$ for $t \in\left[-\omega, \tau_{e}\right)$, where $\tau_{e}$ is the explosion time [51].

Theorem 2. For any given initial value $(S(0), E(0), I(0), Q(0)$, $R(0)) \in \mathbb{R}_{+}^{5}$, system (5) has a unique positive solution $(S(t)$, $E(t), I(t), Q(t), R(t)) \in \mathbb{R}_{+}^{5}$ on $t \geq 0$ a.s.

Proof. The following proof is divided into two parts.

Part I. By Lemma 1, it is easy to see that system (5) has a unique positive local solution $(S(t), E(t), I(t), Q(t), R(t))$ for any given initial value $(S(0), E(0), I(0), Q(0), R(0)) \in \mathbb{R}_{+}^{5}$.

Part II. Now we prove that the positive solution is global, that is, $\tau_{e}=\infty$ a.s. Let $k_{0} \geq 0$ be sufficiently large such that $S(0)$, $E(0), I(0), Q(0)$, and $R(0)$ all lie in $\left[1 / k_{0}, k_{0}\right]$. For each integer $k \geq k_{0}$, let us define the stopping time as follows:

$$
\begin{aligned}
\tau_{k} & =\inf \left\{t \in\left[-\omega, \tau_{e}\right): S(t) \notin\left(\frac{1}{k}, k\right), E(t)\right. \\
& \notin\left(\frac{1}{k}, k\right), I(t) \notin\left(\frac{1}{k}, k\right), Q(t) \notin\left(\frac{1}{k}, k\right) \text { or } R(t) \\
& \left.\notin\left(\frac{1}{k}, k\right)\right\},
\end{aligned}
$$

where we define inf $\emptyset=\infty$ ( $\emptyset$ represents the empty set). Evidently, $\tau_{k}$ is strictly increasing when $k \rightarrow \infty$. Let $\tau_{\infty}=$ $\lim _{k \rightarrow \infty} \tau_{k}$; thus $\tau_{\infty} \leq \tau_{e}$ a.s. So we just need to show that $\tau_{\infty}=\infty$ a.s. If $\tau_{\infty}=\infty$ is untrue, then there exist two constants $T>0$ and $\varsigma \in(0,1)$ such that $\mathbb{P}\left\{\tau_{\infty} \leq T\right\}>\varsigma$. Thus there exists $k_{1} \geq k_{0}\left(k_{1} \in N_{+}\right)$such that

$$
\mathbb{P}\left\{\tau_{k} \leq T\right\} \geq \varsigma, \quad k \geq k_{1} .
$$

Define a $C^{2}$-function $\widehat{V}: \mathbb{R}_{+}^{5} \rightarrow \mathbb{R}_{+}$by

$$
\begin{aligned}
\widehat{V}(S, E, I, Q, R)= & S-1-\ln S+E-1-\ln E+I-1 \\
& -\ln I+Q-1-\ln Q+R-1 \\
& -\ln R .
\end{aligned}
$$

Applying Itô's formula and system (5), we have

$$
\begin{aligned}
\mathrm{d} \widehat{V}= & \mathscr{L} \widehat{V} \mathrm{~d} t+\sigma_{1}(S-1) \mathrm{d} B_{1}(t)+\sigma_{2}(E-1) \mathrm{d} B_{2}(t) \\
& +\sigma_{3}(I-1) \mathrm{d} B_{3}(t)+\sigma_{4}(Q-1) \mathrm{d} B_{4}(t) \\
& +\sigma_{5}(R-1) \mathrm{d} B_{5}(t),
\end{aligned}
$$

where

$$
\begin{aligned}
\mathscr{L} \widehat{V} & =\left(1-\frac{1}{S}\right) \\
\cdot & {\left[\Lambda-\frac{(1-\delta) \beta S I}{N-Q}-\frac{\delta(1-p) \beta S I}{N-Q}-(\delta p+\mu) S\right] } \\
+ & \left(1-\frac{1}{E}\right) \\
+ & {\left[\frac{(1-\delta) \beta S I}{N-Q}+\frac{\delta(1-p) \beta S I}{N-Q}-(\alpha+\mu) E\right] } \\
+ & \left(1-\frac{1}{I}\right)\left[\alpha E-\left(\lambda+\varepsilon_{1}+\gamma+\mu\right) I\right]+\left(1-\frac{1}{Q}\right) \\
+ & {\left[\lambda I-\left(\varphi+\varepsilon_{2}+\mu\right) Q\right]+\left(1-\frac{1}{R}\right) } \\
+ & \frac{1}{2}\left(\sigma_{1}^{2}+\sigma_{2}^{2}+\sigma_{3}^{2}+\sigma_{4}^{2}+\sigma_{5}^{2}\right)=M_{0}, \\
+ & \left.\frac{\varphi Q}{R}+\gamma I+\varphi Q-\mu R\right) \\
+ & \frac{1}{2}\left(\sigma_{1}^{2}+\sigma_{2}^{2}+\sigma_{3}^{2}+\sigma_{4}^{2}+\sigma_{5}^{2}\right)=\Lambda+\delta p+\alpha+\lambda \\
+ & \gamma+\varphi+\varepsilon_{1}+\varepsilon_{2}+5 \mu+\frac{1}{S}(1-\delta p) \beta I \\
+ & \mu\left(S+E+I+R+\sigma_{1}^{2}+\sigma_{2}^{2}+\sigma_{4}^{2}+\sigma_{5}^{2}\right) \leq \Lambda+\delta p+\alpha \\
+ &
\end{aligned}
$$


and here $M_{0}$ is a positive constant. Hence

$$
\begin{aligned}
\mathrm{d} \widehat{V} \leq & M_{0} \mathrm{~d} t+\sigma_{1}(S-1) \mathrm{d} B_{1}(t)+\sigma_{2}(E-1) \mathrm{d} B_{2}(t) \\
& +\sigma_{3}(I-1) \mathrm{d} B_{3}(t)+\sigma_{4}(Q-1) \mathrm{d} B_{4}(t) \\
& +\sigma_{5}(R-1) \mathrm{d} B_{5}(t) .
\end{aligned}
$$

Integrating both sides of (11) from 0 to $\tau_{k} \wedge T$ and then taking the expectation, we have

$$
\begin{aligned}
& \mathbb{E} \widehat{V}\left(S\left(\tau_{k} \wedge T\right), E\left(\tau_{k} \wedge T\right), I\left(\tau_{k} \wedge T\right), Q\left(\tau_{k} \wedge T\right),\right. \\
& \left.R\left(\tau_{k} \wedge T\right)\right) \leq \widehat{V}(S(0), E(0), I(0), Q(0), R(0)) \\
& \quad+\mathbb{E} \int_{0}^{\tau_{k} \wedge T} M_{0} \mathrm{~d} t \leq \widehat{V}(S(0), E(0), I(0), Q(0), R(0)) \\
& \quad+M_{0} T
\end{aligned}
$$

Set $\Omega_{k}=\left\{\tau_{k} \leq T\right\}, k \geq k_{1}$ and by (7) we can get that $P\left(\Omega_{k}\right) \geq$ c. Notice that, for every $\omega \in \Omega_{k}$, there exists $S\left(\tau_{k}, \omega\right), E\left(\tau_{k}, \omega\right)$, $I\left(\tau_{k}, \omega\right), Q\left(\tau_{k}, \omega\right)$, or $R\left(\tau_{k}, \omega\right)$ which equals either $1 / k$ or $k$. Thus

$$
\begin{aligned}
& \widehat{V}\left(S\left(\tau_{k}, \omega\right), E\left(\tau_{k}, \omega\right), I\left(\tau_{k}, \omega\right), Q\left(\tau_{k}, \omega\right), R\left(\tau_{k}, \omega\right)\right) \\
& \quad \geq\left(\frac{1}{k}-1-\ln \frac{1}{k}\right) \wedge(k-1-\ln k)
\end{aligned}
$$

By virtue of (12) and (13), one has

$$
\begin{aligned}
& \widehat{V}(S(0), E(0), I(0), Q(0), R(0))+M_{0} T \\
& \quad \geq \mathbb{E}\left[1 _ { \Omega _ { k } ( \omega ) } \widehat { V } \left(S\left(\tau_{k}, \omega\right), E\left(\tau_{k}, \omega\right), I\left(\tau_{k}, \omega\right),\right.\right. \\
& \left.\left.\quad Q\left(\tau_{k}, \omega\right), R\left(\tau_{k}, \omega\right)\right)\right] \geq \varsigma\left[\left(\frac{1}{k}-1-\ln \frac{1}{k}\right) \wedge(k-1\right. \\
& \quad-\ln k)],
\end{aligned}
$$

and here $1_{\Omega_{k}(\omega)}$ is the indicator function of $\Omega_{k}(\omega)$. Let $k \rightarrow \infty$, which implies

$$
\infty>\widehat{V}(S(0), E(0), I(0), Q(0), R(0))+M_{0} T=\infty
$$

is a contradiction. Obviously, we get that $\tau_{\infty}=\infty$. This completes the proof of Theorem 2 .

\section{Extinction}

In this section, we mainly explore the parameter conditions for extinction of the disease in system (5). Before proving the main results, we first give a useful lemma as follows.
Lemma 3. For any given initial value $(S(0), E(0), I(0)$, $Q(0), R(0)) \in \mathbb{R}_{+}^{5}$, the solution $(S(t), E(t), I(t), Q(t), R(t))$ of the system (5) has the following properties:

$$
\begin{aligned}
& \lim _{t \rightarrow \infty} \frac{S(t)}{t}=0, \\
& \lim _{t \rightarrow \infty} \frac{E(t)}{t}=0, \\
& \lim _{t \rightarrow \infty} \frac{I(t)}{t}=0, \\
& \lim _{t \rightarrow \infty} \frac{Q(t)}{t}=0, \\
& \lim _{t \rightarrow \infty} \frac{R(t)}{t}=0
\end{aligned}
$$

a.s.

Furthermore, when $\mu>(1 / 2)\left(\sigma_{1}^{2} \vee \sigma_{2}^{2} \vee \sigma_{3}^{2} \vee \sigma_{4}^{2} \vee \sigma_{5}^{2}\right)$ holds, then

$$
\begin{aligned}
& \lim _{t \rightarrow \infty} \frac{1}{t} \int_{0}^{t} S(r) \mathrm{d} B_{1}(r)=0, \\
& \lim _{t \rightarrow \infty} \frac{1}{t} \int_{0}^{t} E(r) \mathrm{d} B_{2}(r)=0, \\
& \lim _{t \rightarrow \infty} \frac{1}{t} \int_{0}^{t} I(r) \mathrm{d} B_{3}(r)=0, \\
& \lim _{t \rightarrow \infty} \frac{1}{t} \int_{0}^{t} \mathrm{Q}(r) \mathrm{d} B_{4}(r)=0, \\
& \lim _{t \rightarrow \infty} \frac{1}{t} \int_{0}^{t} R(r) \mathrm{d} B_{5}(r)=0
\end{aligned}
$$

a.s.

Proof. The proof of Lemma 3 is similar to [25, 41]; thus we omit it here.

Theorem 4. Let $\mu>(1 / 2)\left(\sigma_{1}^{2} \vee \sigma_{2}^{2} \vee \sigma_{3}^{2} \vee \sigma_{4}^{2} \vee \sigma_{5}^{2}\right)$. For any given initial value $(S(0), E(0), I(0), Q(0), R(0)) \in \mathbb{R}_{+}^{5}$, if

$$
\begin{aligned}
\widetilde{R}^{*} & :=\frac{2 \alpha(1-\delta p) \beta(\alpha+\mu)}{\left(\lambda+\varepsilon_{1}+\gamma+\mu+\sigma_{3}^{2} / 2\right)(\alpha+\mu)^{2} \wedge\left(\alpha^{2} \sigma_{2}^{2} / 2\right)} \\
& <1
\end{aligned}
$$

holds, then

$$
\lim _{t \rightarrow \infty} E(t)=\lim _{t \rightarrow \infty} I(t)=\lim _{t \rightarrow \infty} Q(t)=0 \quad \text { a.s. }
$$

Moreover,

$$
\begin{aligned}
& \lim _{t \rightarrow \infty}\langle S\rangle=\frac{\Lambda}{\delta p+\mu}=S_{0}, \\
& \lim _{t \rightarrow \infty}\langle R\rangle=\frac{\delta p \Lambda}{\mu(\delta p+\mu)}=R_{0}
\end{aligned}
$$

Proof. Define a differentiable function $V_{0}$ by

$$
V_{0}=\ln [\alpha E(t)+(\alpha+\mu) I(t)] .
$$


From Itô's formula and system (5), we have

$$
\begin{aligned}
\mathrm{d} V_{0}= & \left\{\frac{\alpha(1-\delta p) \beta S I /(S+E+I+R)-(\alpha+\mu)\left(\lambda+\varepsilon_{1}+\gamma+\mu\right) I}{\alpha E+(\alpha+\mu) I}-\frac{\alpha^{2} \sigma_{2}^{2} E^{2}+(\alpha+\mu)^{2} \sigma_{3}^{2} I^{2}}{2[\alpha E+(\alpha+\mu) I]^{2}}\right\} \mathrm{d} t \\
& +\frac{\alpha \sigma_{2} E}{\alpha E+(\alpha+\mu) I} \mathrm{~d} B_{2}(t)+\frac{(\alpha+\mu) \sigma_{3} I}{\alpha E+(\alpha+\mu) I} \mathrm{~d} B_{3}(t) \\
\leq & \left\{\frac{\alpha(1-\delta p) \beta}{\alpha+\mu}-\frac{\left(\lambda+\varepsilon_{1}+\gamma+\mu+\sigma_{3}^{2} / 2\right)(\alpha+\mu)^{2} I^{2}+\left(\alpha^{2} \sigma_{2}^{2} / 2\right) E^{2}}{[\alpha E+(\alpha+\mu) I]^{2}}\right\} \mathrm{d} t+\frac{\alpha \sigma_{2} E}{\alpha E+(\alpha+\mu) I} \mathrm{~d} B_{2}(t) \\
& +\frac{(\alpha+\mu) \sigma_{3} I}{\alpha E+(\alpha+\mu) I} \mathrm{~d} B_{3}(t) \\
\leq & \left\{\frac{\alpha(1-\delta p) \beta}{\alpha+\mu}-\frac{\left(\lambda+\varepsilon_{1}+\gamma+\mu+\sigma_{3}^{2} / 2\right)(\alpha+\mu)^{2} \wedge\left(\alpha^{2} \sigma_{2}^{2} / 2\right)}{2(\alpha+\mu)^{2}}\right\} \mathrm{d} t+\frac{\alpha \sigma_{2} E}{\alpha E+(\alpha+\mu) I} \mathrm{~d} B_{2}(t) \\
& +\frac{(\alpha+\mu) \sigma_{3} I}{\alpha E+(\alpha+\mu) I} \mathrm{~d} B_{3}(t) .
\end{aligned}
$$

Integrating from 0 to $t$ and dividing by $t$ on both sides of (22), we have

$$
\begin{aligned}
& \frac{\ln [\alpha E(t)+(\alpha+\mu) I(t)]}{t} \\
& \leq \frac{\alpha(1-\delta p) \beta}{\alpha+\mu} \\
& \quad-\frac{\left(\lambda+\varepsilon_{1}+\gamma+\mu+\sigma_{3}^{2} / 2\right)(\alpha+\mu)^{2} \wedge\left(\alpha^{2} \sigma_{2}^{2} / 2\right)}{2(\alpha+\mu)^{2}} \\
& +\frac{\ln [\alpha E(0)+(\alpha+\mu) I(0)]}{t} \\
& +\frac{\alpha \sigma_{2}}{t} \int_{0}^{t} \frac{E(r)}{\alpha E(r)+(\alpha+\mu) I(r)} \mathrm{d} B_{2}(r) \\
& +\frac{(\alpha+\mu) \sigma_{3}}{t} \int_{0}^{t} \frac{I(r)}{\alpha E(r)+(\alpha+\mu) I(r)} \mathrm{d} B_{3}(r) .
\end{aligned}
$$

Making use of Lemma 3, we have

$$
\begin{aligned}
\limsup _{t \rightarrow \infty} & \frac{\ln [\alpha E(t)+(\alpha+\mu) I(t)]}{t} \\
\leq & \frac{\alpha(1-\delta p) \beta}{\alpha+\mu} \\
& -\frac{\left(\lambda+\varepsilon_{1}+\gamma+\mu+\sigma_{3}^{2} / 2\right)(\alpha+\mu)^{2} \wedge\left(\alpha^{2} \sigma_{2}^{2} / 2\right)}{2(\alpha+\mu)^{2}}
\end{aligned}
$$$$
<0 \quad \text { a.s. }
$$

which shows that

$$
\begin{gathered}
\lim _{t \rightarrow \infty} E(t)=0, \\
\lim _{t \rightarrow \infty} I(t)=0
\end{gathered}
$$

a.s.

From the fourth equation of system (5), it is easy to get that

$$
\lim _{t \rightarrow \infty} Q(t)=0 \quad \text { a.s. }
$$

Moreover, integrating from 0 to $t$ and dividing by $t$ on both sides of the first equation of system (5) yield

$$
\begin{aligned}
\frac{S(t)-S(0)}{t}= & \Lambda-(1-\delta p) \beta\left\langle\frac{S I}{N-Q}\right\rangle \\
& -(\delta p+\mu)\langle S\rangle+\frac{\sigma_{1}}{t} \int_{0}^{t} S(r) \mathrm{d} B_{1}(r),
\end{aligned}
$$

and considering (25), (26), and Lemma 3, it then follows that

$$
\lim _{t \rightarrow \infty}\langle S\rangle=\frac{\Lambda}{\delta p+\mu}=S_{0} \quad \text { a.s. }
$$

Similarly, we also get

$$
\lim _{t \rightarrow \infty}\langle R\rangle=\frac{\delta p \Lambda}{\mu(\delta p+\mu)}=R_{0} \quad \text { a.s. }
$$

The proof of Theorem 4 is complete.

\section{Stationary Distribution and Ergodicity}

Ergodicity is a significant property in a population system. Recently, it attracts deep research interest of numerous 
scholars $[52,53]$. In this section, based on the theory of Hasminskii et al. [54] and the Lyapunov analysis methods, we study the conditions of the existence of an ergodic stationary distribution.

Assume $X(t)$ as a time-homogeneous Markov process in $\mathbb{E}_{n} \subset \mathbb{R}^{n}$, which is described by the stochastic differential equation

$$
\mathrm{d} X(t)=b(X) \mathrm{d} t+\sum_{\eta=1}^{n} \sigma_{\eta}(X) \mathrm{d} B_{\eta}(t)
$$

and here $\mathbb{E}_{n}$ stands for a $n$-dimensional Euclidean space. The diffusion matrix takes the following form:

$$
\widetilde{A}(x)=\left(a_{i j}(x)\right), \quad a_{i j}(x)=\sum_{\eta=1}^{n} \sigma_{\eta}^{i}(x) \sigma_{\eta}^{j}(x) .
$$

Assumption 5. Assume that there is a bounded domain $U \subset$ $\mathbb{E}_{n}$ with regular boundary $\Gamma$ such that $\bar{U} \subset \mathbb{E}_{n}(\bar{U}$ is the closure of $U$ ), satisfying the following properties:

(i) In the domain $U$ and some neighborhood thereof, the smallest eigenvalue of the diffusion matrix $\widetilde{A}(x)$ is bounded away from zero.

(ii) If $x \in \mathbb{E}_{n} \backslash U$, the mean time $\tau$ at which a path issuing from $x$ reaches the set $U$ is finite, and $\sup _{x \in \Theta} \mathbb{E}_{x} \tau<\infty$ for every compact subset $\Theta \subset \mathbb{E}_{n}$.

Lemma 6 (see [54]). When Assumption 5 holds, then the Markov process $X(t)$ has a stationary distribution $\pi(\cdot)$. Furthermore, when $f(\cdot)$ is a function integrable with respect to the measure $\pi$, then

$$
\mathbb{P}_{x}\left\{\lim _{T \rightarrow \infty} \frac{1}{T} \int_{0}^{T} f(X(t)) \mathrm{d} t=\int_{\mathbb{E}_{n}} f(x) \pi(\mathrm{d} x)\right\}=1
$$

for all $x \in \mathbb{E}_{n}$.

Remark 7. To demonstrate Assumption 5(i) [55], it suffices to demonstrate that $F$ is uniformly elliptical in any bounded domain $H$; here

$$
F u=b(x) u_{x}+\frac{1}{2} \operatorname{trace}\left(A(x) u_{x x}\right)
$$

namely, there exists a positive number $Z$ such that

$$
\sum_{i, j=1}^{n} a_{i j}(x) \xi_{i} \xi_{j} \geq Z|\xi|^{2}, \quad x \in \bar{H}, \quad \xi \in \mathbb{R}^{n}
$$

To verify Assumption 5(ii) [56], it suffices to demonstrate that there exist some neighborhood $U$ and a nonnegative $C^{2}$ function $V$ such that $\forall x \in \mathbb{E}_{n} \backslash U, L V(x)<0$.

Using Lemma 6, we can get the following main results.
Theorem 8. For any given initial value $(S(0), E(0), I(0)$, $Q(0), R(0)) \in \mathbb{R}_{+}^{5}$. If

$$
\begin{aligned}
& R^{*} \\
& :=\frac{\mu(1-\delta p) \beta \alpha}{\left(\delta p+\mu+\sigma_{1}^{2} / 2\right)\left(\alpha+\mu+\sigma_{2}^{2} / 2\right)\left(\lambda+\varepsilon_{1}+\gamma+\mu+\sigma_{3}^{2} / 2\right)} \\
& >1
\end{aligned}
$$

holds, then system (5) has a unique stationary distribution $\pi(\cdot)$ and it has ergodic property.

Proof. Define a $C^{2}$-function $\bar{V}: \mathbb{R}_{+}^{5} \rightarrow \mathbb{R}$ by

$$
\begin{aligned}
\bar{V}(S, E, I, Q, R) \\
=\Upsilon\left(S+E+I+Q+R-a_{1} \ln S-a_{2} \ln E-a_{3} \ln I\right) \\
\quad+\frac{1}{\varrho+1}(S+E+I+Q+R)^{\varrho+1}-\ln S-\ln E \\
\quad-\ln Q-\ln R+(S+E+I+Q+R) \\
:=\Upsilon V_{1}+V_{2}+V_{3}+V_{4}+V_{5}+V_{6}+V_{7},
\end{aligned}
$$

and here $\varrho$ and $a_{i}(i=1,2,3)$ are positive constants satisfying the following conditions:

$$
\begin{aligned}
& 0<\varrho<\frac{2 \mu}{\sigma_{1}^{2} \vee \sigma_{2}^{2} \vee \sigma_{3}^{2} \vee \sigma_{4}^{2} \vee \sigma_{5}^{2}}, \\
& a_{1}=\frac{\Lambda}{\delta p+\mu+\sigma_{1}^{2} / 2}, \\
& a_{2}=\frac{\Lambda}{\alpha+\mu+\sigma_{2}^{2} / 2}, \\
& a_{3}=\frac{\Lambda}{\lambda+\varepsilon_{1}+\gamma+\mu+\sigma_{3}^{2} / 2},
\end{aligned}
$$

and we take $\Upsilon>0$ large enough such that

$$
-\Upsilon \phi+M \leq-2
$$

here

$$
\begin{aligned}
\phi:= & 4 \Lambda\left[\left(R^{*}\right)^{1 / 4}-1\right], \\
M:= & \Lambda+\Gamma+\delta p+\alpha+\varphi+\varepsilon_{2}+4 \mu+\frac{\sigma_{1}^{2}}{2}+\frac{\sigma_{2}^{2}}{2}+\frac{\sigma_{4}^{2}}{2} \\
& +\frac{\sigma_{5}^{2}}{2} .
\end{aligned}
$$

Obviously,

$$
\lim _{w \rightarrow,(S, E, I, Q, R) \in \mathbb{R}_{+}^{5} \backslash U_{w}} \bar{V}(S, E, I, Q, R)=\infty,
$$

and here $U_{w}=(1 / w, w) \times(1 / w, w) \times(1 / w, w) \times(1 / w, w) \times$ $(1 / w, w)$. Since $\bar{V}(S, E, I, Q, R)$ is a continuous function, then there exists a unique point $\left(\underline{S^{*}}, \underline{E^{*}}, \underline{I^{*}}, Q^{*}, \underline{R^{*}}\right)$ in $\mathbb{R}_{+}^{5}$ which is the minimum point of $\bar{V}(S, E, I, Q, R)$. Therefore let us construct a positive-definite $C^{2}$-function $V: \mathbb{R}_{+}^{5} \rightarrow \mathbb{R}_{+}$by

$$
\begin{aligned}
V(S, E, I, Q, R)= & \bar{V}(S, E, I, Q, R) \\
& -\bar{V}\left(\underline{S^{*}}, \underline{E^{*}}, \underline{I^{*}}, \underline{Q^{*}}, \underline{R^{*}}\right) .
\end{aligned}
$$


From Itô's formula, we have

$$
\begin{aligned}
\mathscr{L} V_{1}= & -\mu(S+E+I+Q+R)-\frac{a_{1} \Lambda}{S}-\frac{a_{2}(1-\delta) \beta S I}{E(N-Q)}-\frac{a_{2} \delta(1-p) \beta S I}{E(N-Q)}-\frac{a_{3} \alpha E}{I}+\frac{a_{1}(1-\delta) \beta I}{N-Q}+\frac{a_{1} \delta(1-p) \beta I}{N-Q} \\
& +\Lambda+a_{1}\left(\delta p+\mu+\frac{\sigma_{1}^{2}}{2}\right)+a_{2}\left(\alpha+\mu+\frac{\sigma_{2}^{2}}{2}\right)+a_{3}\left(\lambda+\varepsilon_{1}+\gamma+\mu+\frac{\sigma_{3}^{2}}{2}\right)-\varepsilon_{1} I-\varepsilon_{2} Q \\
\leq & -\mu(S+E+I+Q+R)-\frac{a_{1} \Lambda}{S}-\frac{a_{2}(1-\delta p) \beta S I}{E(S+E+I+Q+R)}-\frac{a_{3} \alpha E}{I}+\frac{a_{1}(1-\delta p) \beta I}{S+E+I+R}+\Lambda+a_{1}\left(\delta p+\mu+\frac{\sigma_{1}^{2}}{2}\right) \\
& +a_{2}\left(\alpha+\mu+\frac{\sigma_{2}^{2}}{2}\right)+a_{3}\left(\lambda+\varepsilon_{1}+\gamma+\mu+\frac{\sigma_{3}^{2}}{2}\right) \leq-4\left(a_{1} a_{2} a_{3} \Lambda \mu(1-\delta p) \beta \alpha\right)^{1 / 4}+\frac{a_{1}(1-\delta p) \beta I}{S+E+I+R}+4 \Lambda \\
= & -4 \Lambda\left[\left(\frac{\mu(1-\delta p) \beta \alpha}{\left(\delta p+\mu+\sigma_{1}^{2} / 2\right)\left(\alpha+\mu+\sigma_{2}^{2} / 2\right)\left(\lambda+\varepsilon_{1}+\gamma+\mu+\sigma_{3}^{2} / 2\right)}\right)^{1 / 4}-1\right]+\frac{a_{1}(1-\delta p) \beta I}{S+E+I+R} \\
= & -4 \Lambda\left[\left(R^{*}\right)^{1 / 4}-1\right]+\frac{a_{1}(1-\delta p) \beta I}{S+E+I+R}=-\phi+\frac{a_{1}(1-\delta p) \beta I}{S+E+I+R} .
\end{aligned}
$$

Similarly,

$$
\begin{aligned}
\mathscr{L} V_{2} & =(S+E+I+Q+R)^{\varrho} \\
\cdot & {\left[\Lambda-\mu(S+E+I+Q+R)-\varepsilon_{1} I-\varepsilon_{2} Q\right] } \\
+ & \frac{\varrho(S+E+I+Q+R)^{\varrho-1}}{2} \\
\times & \left(\sigma_{1}^{2} S^{2}+\sigma_{2}^{2} E^{2}+\sigma_{3}^{2} I^{2}+\sigma_{4}^{2} Q^{2}+\sigma_{5}^{2} R^{2}\right) \\
\leq & \Lambda(S+E+I+Q+R)^{\varrho} \\
- & {\left[\mu-\frac{\varrho}{2}\left(\sigma_{1}^{2} \vee \sigma_{2}^{2} \vee \sigma_{3}^{2} \vee \sigma_{4}^{2} \vee \sigma_{5}^{2}\right)\right] } \\
\cdot & (S+E+I+Q+R)^{\varrho+1} \leq \Gamma \\
- & \frac{1}{2}\left[\mu-\frac{\varrho}{2}\left(\sigma_{1}^{2} \vee \sigma_{2}^{2} \vee \sigma_{3}^{2} \vee \sigma_{4}^{2} \vee \sigma_{5}^{2}\right)\right] \\
\cdot & (S+E+I+Q+R)^{\varrho+1} \leq \Gamma \\
- & \frac{1}{2}\left[\mu-\frac{\varrho}{2}\left(\sigma_{1}^{2} \vee \sigma_{2}^{2} \vee \sigma_{3}^{2} \vee \sigma_{4}^{2} \vee \sigma_{5}^{2}\right)\right] \\
\cdot & \left(S^{\varrho+1}+E^{\varrho+1}+I^{\varrho+1}+Q^{\varrho+1}+R^{\varrho+1}\right),
\end{aligned}
$$

and here

$$
\begin{aligned}
\Gamma= & \sup _{(S, E, I, Q, R) \in \mathbb{R}_{+}^{5}}\left\{\Lambda(S+E+I+Q+R)^{\varrho}\right. \\
& -\frac{1}{2}\left[\mu-\frac{\varrho}{2}\left(\sigma_{1}^{2} \vee \sigma_{2}^{2} \vee \sigma_{3}^{2} \vee \sigma_{4}^{2} \vee \sigma_{5}^{2}\right)\right] \\
& \left.\times(S+E+I+Q+R)^{\varrho+1}\right\}<\infty .
\end{aligned}
$$

We also have

$$
\begin{aligned}
& \mathscr{L} V_{3}=-\frac{\Lambda}{S}+\frac{(1-\delta p) \beta I}{S+E+I+R}+\delta p+\mu+\frac{\sigma_{1}^{2}}{2}, \\
& \mathscr{L} V_{4} \leq-\frac{(1-\delta p) \beta S I}{E(S+E+I+Q+R)}+\alpha+\mu+\frac{\sigma_{2}^{2}}{2}, \\
& \mathscr{L} V_{5}=-\frac{\lambda I}{Q}+\varphi+\varepsilon_{2}+\mu+\frac{\sigma_{4}^{2}}{2}, \\
& \mathscr{L} V_{6}=-\frac{\delta p S+\gamma I+\varphi Q}{R}+\mu+\frac{\sigma_{5}^{2}}{2}, \\
& \mathscr{L} V_{7} \leq \Lambda-\mu(S+E+I+Q+R) .
\end{aligned}
$$

Therefore,

$$
\begin{aligned}
\mathscr{L} V & \leq-\Upsilon \phi+\frac{\Upsilon a_{1}(1-\delta p) \beta I}{S+E+I+R}+\frac{(1-\delta p) \beta I}{S+E+I+R} \\
- & \frac{1}{2}\left[\mu-\frac{\varrho}{2}\left(\sigma_{1}^{2} \vee \sigma_{2}^{2} \vee \sigma_{3}^{2} \vee \sigma_{4}^{2} \vee \sigma_{5}^{2}\right)\right] \\
& \times\left(S^{\varrho+1}+E^{\varrho+1}+I^{\varrho+1}+Q^{\varrho+1}+R^{\varrho+1}\right)-\frac{\Lambda}{S} \\
& -\frac{(1-\delta p) \beta S I}{E(S+E+I+Q+R)}-\mu(S+E+I+Q+R) \\
& -\frac{\lambda I}{Q}-\frac{\delta p S+\gamma I+\varphi Q}{R}+\Lambda+\Gamma+\delta p+\alpha+\varphi+\varepsilon_{2} \\
+ & 4 \mu+\frac{\sigma_{1}^{2}}{2}+\frac{\sigma_{2}^{2}}{2}+\frac{\sigma_{4}^{2}}{2}+\frac{\sigma_{5}^{2}}{2} \leq-\Upsilon \phi \\
+ & \frac{\left(\Upsilon a_{1}+1\right)(1-\delta p) \beta I}{S+E+I+R}
\end{aligned}
$$




$$
\begin{aligned}
& -\frac{1}{2}\left[\mu-\frac{\varrho}{2}\left(\sigma_{1}^{2} \vee \sigma_{2}^{2} \vee \sigma_{3}^{2} \vee \sigma_{4}^{2} \vee \sigma_{5}^{2}\right)\right] \\
& \cdot\left(S^{\varrho+1}+E^{\varrho+1}+I^{\varrho+1}+Q^{\varrho+1}+R^{\varrho+1}\right)-\frac{\Lambda}{S} \\
& -2\left(\frac{\mu(1-\delta p) \beta S I}{E}\right)^{1 / 2}-\frac{\lambda I}{Q}-\frac{\delta p S+\gamma I+\varphi Q}{R}
\end{aligned}
$$$$
+M
$$

where $M=\Lambda+\Gamma+\delta p+\alpha+\varphi+\varepsilon_{2}+4 \mu+\sigma_{1}^{2} / 2+\sigma_{2}^{2} / 2+\sigma_{4}^{2} / 2+\sigma_{5}^{2} / 2$. Next let us consider the following compact subset $D$ :

$$
\begin{aligned}
D & =\left\{\epsilon \leq S \leq \frac{1}{\epsilon}, \epsilon^{4} \leq E \leq \frac{1}{\epsilon^{4}}, \epsilon^{2} \leq I \leq \frac{1}{\epsilon^{2}}, \epsilon^{3} \leq Q\right. \\
& \left.\leq \frac{1}{\epsilon^{3}}, \epsilon^{4} \leq R \leq \frac{1}{\epsilon^{4}}\right\},
\end{aligned}
$$

and here $\epsilon$ is a sufficiently small constant satisfying the following conditions:

$$
\begin{aligned}
& -\frac{\Lambda}{\epsilon}+\left(\Upsilon a_{1}+1\right)(1-\delta p) \beta+M \leq-1, \\
& -2\left(\frac{\mu(1-\delta p) \beta}{\epsilon}\right)^{1 / 2}+\left(\Upsilon a_{1}+1\right)(1-\delta p) \beta \\
& +M \leq-1 \text {, } \\
& -\Upsilon \phi+\left(\Upsilon a_{1}+1\right)(1-\delta p) \beta \epsilon+M \leq-1, \\
& -\frac{\lambda}{\epsilon}+\left(\Upsilon a_{1}+1\right)(1-\delta p) \beta+M \leq-1, \\
& -\frac{\delta p}{\epsilon^{3}}-\frac{\gamma}{\epsilon^{2}}-\frac{\varphi}{\epsilon}+\left(\Upsilon a_{1}+1\right)(1-\delta p) \beta+M \leq-1, \\
& -\frac{1}{2}\left[\mu-\frac{\varrho}{2}\left(\sigma_{1}^{2} \vee \sigma_{2}^{2} \vee \sigma_{3}^{2} \vee \sigma_{4}^{2} \vee \sigma_{5}^{2}\right)\right] \frac{1}{\epsilon^{\varrho+1}} \\
& +\left(\Upsilon a_{1}+1\right)(1-\delta p) \beta+M \leq-1, \\
& -\frac{1}{2}\left[\mu-\frac{\varrho}{2}\left(\sigma_{1}^{2} \vee \sigma_{2}^{2} \vee \sigma_{3}^{2} \vee \sigma_{4}^{2} \vee \sigma_{5}^{2}\right)\right] \frac{1}{\epsilon^{4(\varrho+1)}} \\
& +\left(\Upsilon a_{1}+1\right)(1-\delta p) \beta+M \leq-1, \\
& -\frac{1}{2}\left[\mu-\frac{\varrho}{2}\left(\sigma_{1}^{2} \vee \sigma_{2}^{2} \vee \sigma_{3}^{2} \vee \sigma_{4}^{2} \vee \sigma_{5}^{2}\right)\right] \frac{1}{\epsilon^{2(\varrho+1)}} \\
& +\left(\Upsilon a_{1}+1\right)(1-\delta p) \beta+M \leq-1, \\
& -\frac{1}{2}\left[\mu-\frac{\varrho}{2}\left(\sigma_{1}^{2} \vee \sigma_{2}^{2} \vee \sigma_{3}^{2} \vee \sigma_{4}^{2} \vee \sigma_{5}^{2}\right)\right] \frac{1}{\epsilon^{3(\varrho+1)}} \\
& +\left(\Upsilon a_{1}+1\right)(1-\delta p) \beta+M \leq-1 \text {. }
\end{aligned}
$$

Then

$$
\begin{aligned}
& \mathbb{R}_{+}^{5} \backslash D= D_{1} \cup D_{2} \cup D_{3} \cup D_{4} \cup D_{5} \cup D_{6} \cup D_{7} \cup D_{8} \\
& \cup D_{9} \cup D_{10}
\end{aligned}
$$

with

$$
\begin{aligned}
D_{1} & =\left\{(S, E, I, Q, R) \in \mathbb{R}_{+}^{5} \mid 0<S<\epsilon\right\}, \\
D_{2} & =\left\{(S, E, I, Q, R) \in \mathbb{R}_{+}^{5} \mid S \geq \epsilon, I \geq \epsilon^{2}, 0<E<\epsilon^{4}\right\}, \\
D_{3} & =\left\{(S, E, I, Q, R) \in \mathbb{R}_{+}^{5} \mid S \geq \epsilon, 0<I<\epsilon^{2}\right\}, \\
D_{4} & =\left\{(S, E, I, Q, R) \in \mathbb{R}_{+}^{5} \mid I \geq \epsilon^{2}, 0<Q<\epsilon^{3}\right\}, \\
D_{5} & =\left\{(S, E, I, Q, R) \in \mathbb{R}_{+}^{5} \mid S \geq \epsilon, I \geq \epsilon^{2}, Q \geq \epsilon^{3}, 0<R\right. \\
& <\epsilon\}, \\
D_{6} & =\left\{(S, E, I, Q, R) \in \mathbb{R}_{+}^{5} \mid S>\frac{1}{\epsilon}\right\}, \\
D_{7} & =\left\{(S, E, I, Q, R) \in \mathbb{R}_{+}^{5} \mid E>\frac{1}{\epsilon^{4}}\right\}, \\
D_{8} & =\left\{(S, E, I, Q, R) \in \mathbb{R}_{+}^{5} \mid I>\frac{1}{\epsilon^{2}}\right\}, \\
D_{9} & =\left\{(S, E, I, Q, R) \in \mathbb{R}_{+}^{5} \mid Q>\frac{1}{\epsilon^{3}}\right\}, \\
D_{10} & =\left\{(S, E, I, Q, R) \in \mathbb{R}_{+}^{5} \mid R>\frac{1}{\epsilon^{4}}\right\} .
\end{aligned}
$$

Now we analyze the negativity of $\mathscr{L} V$ for any $(S, E, I, Q, R) \in$ $\mathbb{R}_{+}^{5} \backslash D$.

Case I. If $(S, I, Q, R) \in D_{1},(46)$ and (48) derive that

$$
\begin{aligned}
\mathscr{L} V & \leq-\frac{\Lambda}{S}+\frac{\left(\Upsilon a_{1}+1\right)(1-\delta p) \beta I}{S+E+I+R}+M \\
& \leq-\frac{\Lambda}{\epsilon}+\left(\Upsilon a_{1}+1\right)(1-\delta p) \beta+M \leq-1
\end{aligned}
$$

Case II. If $(S, I, Q, R) \in D_{2}$, (46) and (49) imply that

$$
\begin{aligned}
\mathscr{L} V \leq & -2\left(\frac{\mu(1-\delta p) \beta S I}{E}\right)^{1 / 2} \\
& +\frac{\left(\Upsilon a_{1}+1\right)(1-\delta p) \beta I}{S+E+I+R}+M \\
\leq & -2\left(\frac{\mu(1-\delta p) \beta}{\epsilon}\right)^{1 / 2}+\left(\Upsilon a_{1}+1\right)(1-\delta p) \beta \\
& +M \leq-1 .
\end{aligned}
$$

Case III. If $(S, I, Q, R) \in D_{3}$, it follows from (46) and (50) that

$$
\begin{aligned}
\mathscr{L} V & \leq-\Upsilon \phi+\frac{\left(\Upsilon a_{1}+1\right)(1-\delta p) \beta I}{S+E+I+R}+M \\
& \leq-\Upsilon \phi+\frac{\left(\Upsilon a_{1}+1\right)(1-\delta p) \beta I}{S}+M \\
& \leq-\Upsilon \phi+\left(\Upsilon a_{1}+1\right)(1-\delta p) \beta \epsilon+M \leq-1 .
\end{aligned}
$$


Case IV. If $(S, I, Q, R) \in D_{4},(46)$ and (51) yield that

$$
\begin{aligned}
\mathscr{L} V & \leq-\frac{\lambda I}{Q}+\frac{\left(\Upsilon a_{1}+1\right)(1-\delta p) \beta I}{S+E+I+R}+M \\
& \leq-\frac{\lambda}{\epsilon}+\left(\Upsilon a_{1}+1\right)(1-\delta p) \beta+M \leq-1 .
\end{aligned}
$$

Case V. If $(S, I, Q, R) \in D_{5}$, it follows from (46) and (52) that

$$
\begin{aligned}
\mathscr{L} V \leq & -\frac{\delta p S+\gamma I+\varphi Q}{R}+\frac{\left(\Upsilon a_{1}+1\right)(1-\delta p) \beta I}{S+E+I+R} \\
& +M \\
\leq & -\frac{\delta p}{\epsilon^{3}}-\frac{\gamma}{\epsilon^{2}}-\frac{\varphi}{\epsilon}+\left(\Upsilon a_{1}+1\right)(1-\delta p) \beta+M \\
\leq & -1 .
\end{aligned}
$$

Case VI. If $(S, I, Q, R) \in D_{6},(46)$ and (53) lead to

$$
\begin{aligned}
\mathscr{L} V \leq & -\frac{1}{2}\left[\mu-\frac{\varrho}{2}\left(\sigma_{1}^{2} \vee \sigma_{2}^{2} \vee \sigma_{3}^{2} \vee \sigma_{4}^{2} \vee \sigma_{5}^{2}\right)\right] S^{\varrho+1} \\
& +\frac{\left(\Upsilon a_{1}+1\right)(1-\delta p) \beta I}{S+E+I+R}+M \\
\leq & -\frac{1}{2}\left[\mu-\frac{\varrho}{2}\left(\sigma_{1}^{2} \vee \sigma_{2}^{2} \vee \sigma_{3}^{2} \vee \sigma_{4}^{2} \vee \sigma_{5}^{2}\right)\right] \frac{1}{\epsilon^{\varrho+1}} \\
& +\left(\Upsilon a_{1}+1\right)(1-\delta p) \beta+M \leq-1 .
\end{aligned}
$$

Case VII. If $(S, I, Q, R) \in D_{7},(46)$ and (54) derive that

$$
\begin{aligned}
\mathscr{L} V \leq & -\frac{1}{2}\left[\mu-\frac{\varrho}{2}\left(\sigma_{1}^{2} \vee \sigma_{2}^{2} \vee \sigma_{3}^{2} \vee \sigma_{4}^{2} \vee \sigma_{5}^{2}\right)\right] E^{\varrho+1} \\
& +\frac{\left(\Upsilon a_{1}+1\right)(1-\delta p) \beta I}{S+E+I+R}+M \\
\leq & -\frac{1}{2}\left[\mu-\frac{\varrho}{2}\left(\sigma_{1}^{2} \vee \sigma_{2}^{2} \vee \sigma_{3}^{2} \vee \sigma_{4}^{2} \vee \sigma_{5}^{2}\right)\right] \frac{1}{\epsilon^{4(\varrho+1)}} \\
& +\left(\Upsilon a_{1}+1\right)(1-\delta p) \beta+M \leq-1 .
\end{aligned}
$$

Case VIII. If $(S, I, Q, R) \in D_{8}$, it follows from (46) and (55) that

$$
\begin{aligned}
\mathscr{L} V \leq & -\frac{1}{2}\left[\mu-\frac{\varrho}{2}\left(\sigma_{1}^{2} \vee \sigma_{2}^{2} \vee \sigma_{3}^{2} \vee \sigma_{4}^{2} \vee \sigma_{5}^{2}\right)\right] I^{\varrho+1} \\
& +\frac{\left(\Upsilon a_{1}+1\right)(1-\delta p) \beta I}{S+E+I+R}+M \\
\leq & -\frac{1}{2}\left[\mu-\frac{\varrho}{2}\left(\sigma_{1}^{2} \vee \sigma_{2}^{2} \vee \sigma_{3}^{2} \vee \sigma_{4}^{2} \vee \sigma_{5}^{2}\right)\right] \frac{1}{\epsilon^{2(\varrho+1)}} \\
& +\left(\Upsilon a_{1}+1\right)(1-\delta p) \beta+M \leq-1 .
\end{aligned}
$$

Case IX. If $(S, I, Q, R) \in D_{9},(46)$ and (56) derive that

$$
\begin{aligned}
\mathscr{L} V \leq & -\frac{1}{2}\left[\mu-\frac{\varrho}{2}\left(\sigma_{1}^{2} \vee \sigma_{2}^{2} \vee \sigma_{3}^{2} \vee \sigma_{4}^{2} \vee \sigma_{5}^{2}\right)\right] Q^{\varrho+1} \\
& +\frac{\left(\Upsilon a_{1}+1\right)(1-\delta p) \beta I}{S+E+I+R}+M \\
\leq & -\frac{1}{2}\left[\mu-\frac{\varrho}{2}\left(\sigma_{1}^{2} \vee \sigma_{2}^{2} \vee \sigma_{3}^{2} \vee \sigma_{4}^{2} \vee \sigma_{5}^{2}\right)\right] \frac{1}{\epsilon^{3(\varrho+1)}} \\
& +\left(\Upsilon a_{1}+1\right)(1-\delta p) \beta+M \leq-1 .
\end{aligned}
$$

Case X. If $(S, I, Q, R) \in D_{10}$, it follows from (46) and (54) that

$$
\begin{aligned}
\mathscr{L} V \leq & -\frac{1}{2}\left[\mu-\frac{\varrho}{2}\left(\sigma_{1}^{2} \vee \sigma_{2}^{2} \vee \sigma_{3}^{2} \vee \sigma_{4}^{2} \vee \sigma_{5}^{2}\right)\right] R^{\varrho+1} \\
& +\frac{\left(\Upsilon a_{1}+1\right)(1-\delta p) \beta I}{S+E+I+R}+M \\
\leq & -\frac{1}{2}\left[\mu-\frac{\varrho}{2}\left(\sigma_{1}^{2} \vee \sigma_{2}^{2} \vee \sigma_{3}^{2} \vee \sigma_{4}^{2} \vee \sigma_{5}^{2}\right)\right] \frac{1}{\epsilon^{4(\varrho+1)}} \\
& +\left(\Upsilon a_{1}+1\right)(1-\delta p) \beta+M \leq-1 .
\end{aligned}
$$

Clearly, from the discussion of the above ten cases, one sees that, for a sufficiently small $\epsilon$,

$$
\mathscr{L} V \leq-1 \quad \forall(S, E, I, Q, R) \in \mathbb{R}_{+}^{5} \backslash D,
$$

which shows that Assumption 5(ii) is satisfied. In addition, the diffusion matrix of system (5) takes the following form:

$$
\widetilde{A}=\left(\begin{array}{ccccc}
\sigma_{1}^{2} S^{2} & 0 & 0 & 0 & 0 \\
0 & \sigma_{2}^{2} E^{2} & 0 & 0 & 0 \\
0 & 0 & \sigma_{3}^{2} I^{2} & 0 & 0 \\
0 & 0 & 0 & \sigma_{4}^{2} Q^{2} & 0 \\
0 & 0 & 0 & 0 & \sigma_{5}^{2} R^{2}
\end{array}\right)
$$

There exists a positive number

$$
Z=\min _{(S, E, I, Q, R) \in \bar{D}}\left\{\sigma_{1}^{2} S^{2}, \sigma_{2}^{2} E^{2}, \sigma_{3}^{2} I^{2}, \sigma_{4}^{2} Q^{2}, \sigma_{5}^{2} R^{2}\right\}
$$

such that

$$
\begin{aligned}
\sum_{i, j=1}^{5} a_{i j} \xi_{i} \xi_{j}= & \sigma_{1}^{2} S^{2} \xi_{1}^{2}+\sigma_{2}^{2} E^{2} \xi_{2}^{2}+\sigma_{3}^{2} I^{2} \xi_{3}^{2}+\sigma_{4}^{2} Q^{2} \xi_{4}^{2} \\
& +\sigma_{5}^{2} R^{2} \xi_{5}^{2} \geq Z|\xi|^{2}
\end{aligned}
$$

$$
(S, E, I, Q, R) \in \bar{D}, \xi \in \mathbb{R}^{5},
$$

which shows that Assumption 5(i) is satisfied. Consequently, system (5) has a unique stationary distribution $\pi(\cdot)$ and it has ergodic property. The proof of Theorem 8 is complete.

Remark 9. From Theorem 8, we see that if $R^{*}>1$ holds, then system (5) has a unique ergodic stationary distribution. It is worthwhile noting that if $\sigma_{i}=0(i=1,2,3,4,5)$, the expression of $R^{*}$ coincides with the basic reproduction number $R_{1}$ of system (3). This shows that we generalize the results of system (3). For another, this theorem also shows that the disease can resist a small environmental noise to maintain the original persistence. 


\section{Conclusions and Simulations}

This paper studies the stochastic SEIQR epidemic model with quarantine-adjusted incidence and imperfect vaccination and obtains two thresholds which govern the extinction and the spread of the epidemic disease. Firstly, the existence of a unique positive solution of system (5) with any positive initial value is proved. Then, from Theorems 4 and 8 , the sufficient conditions for extinction of the disease and existence of ergodic stationary distribution of the stochastic system are derived by using the theory of Hasminskii and the Lyapunov analysis methods, which means the infectious disease is prevalent. This implies that the stochastic disturbance is conducive to epidemic diseases control. Now we summarize the main conclusions as follows:

(I) When $\mu>(1 / 2)\left(\sigma_{1}^{2} \vee \sigma_{2}^{2} \vee \sigma_{3}^{2} \vee \sigma_{4}^{2} \vee \sigma_{5}^{2}\right)$ and $\widetilde{R}^{*}=$ $(2 \alpha(1-\delta p) \beta(\alpha+\mu)) /\left(\left(\lambda+\varepsilon_{1}+\gamma+\mu+\sigma_{3}^{2} / 2\right)(\alpha+\mu)^{2} \wedge\right.$ $\left.\left(\alpha^{2} \sigma_{2}^{2} / 2\right)\right)<1$ hold, then the infected individuals go to extinction almost surely.

(II) When $R^{*}=(\mu(1-\delta p) \beta \alpha) /\left(\left(\delta p+\mu+\sigma_{1}^{2} / 2\right)(\alpha+\mu+\right.$ $\left.\left.\sigma_{2}^{2} / 2\right)\left(\lambda+\varepsilon_{1}+\gamma+\mu+\sigma_{3}^{2} / 2\right)\right)>1$ holds, then system (5) has a unique stationary distribution $\pi(\cdot)$ and it has ergodic property.

To illustrate the results of the above theorems, we next carry out some numerical simulations by the Matlab software. Let us consider the following discretization equations of system (5):

$$
\begin{aligned}
& S_{k+1}=S_{k}+\left[\Lambda-\frac{(1-\delta) \beta S_{k} I_{k}}{S_{k}+E_{k}+I_{k}+R_{k}}\right. \\
& \left.-\frac{\delta(1-p) \beta S_{k} I_{k}}{S_{k}+E_{k}+I_{k}+R_{k}}-(\delta p+\mu) S_{k}\right] \Delta t \\
& +\sigma_{1} S_{k} \sqrt{\Delta t} \zeta_{1, k}+\frac{\sigma_{1}^{2}}{2} S_{k}\left(\zeta_{1, k}^{2}-1\right) \Delta t, \\
& E_{k+1}=E_{k}+\left[\frac{(1-\delta) \beta S_{k} I_{k}}{S_{k}+E_{k}+I_{k}+R_{k}}+\frac{\delta(1-p) \beta S_{k} I_{k}}{S_{k}+E_{k}+I_{k}+R_{k}}\right. \\
& \left.-(\alpha+\mu) E_{k}\right] \Delta t+\sigma_{2} E_{k} \sqrt{\Delta t} \zeta_{2, k}+\frac{\sigma_{2}^{2}}{2} E_{k}\left(\zeta_{2, k}^{2}-1\right) \\
& \text { - } \Delta t \\
& I_{k+1}=I_{k}+\left[\alpha E_{k}-\left(\lambda+\varepsilon_{1}+\gamma+\mu\right) I_{k}\right] \Delta t \\
& +\sigma_{3} I_{k} \sqrt{\Delta t} \zeta_{3, k}+\frac{\sigma_{3}^{2}}{2} I_{k}\left(\zeta_{3, k}^{2}-1\right) \Delta t, \\
& Q_{k+1}=Q_{k}+\left[\lambda I_{k}-\left(\varphi+\varepsilon_{2}+\mu\right) Q_{k}\right] \Delta t \\
& +\sigma_{4} Q_{k} \sqrt{\Delta t} \zeta_{4, k}+\frac{\sigma_{4}^{2}}{2} Q_{k}\left(\zeta_{4, k}^{2}-1\right) \Delta t \\
& R_{k+1}=R_{k}+\left(\delta p S_{k}+\gamma I_{k}+\varphi Q_{k}-\mu R_{k}\right) \Delta t \\
& +\sigma_{5} R_{k} \sqrt{\Delta t} \zeta_{5, k}+\frac{\sigma_{5}^{2}}{2} R_{k}\left(\zeta_{5, k}^{2}-1\right) \Delta t,
\end{aligned}
$$

and here $\zeta_{i, k}(i=1,2,3,4,5 ; k=1,2, \ldots, n)$ stands for $N(0,1)$ distributed independent random variables and time increment $\Delta t>0$.

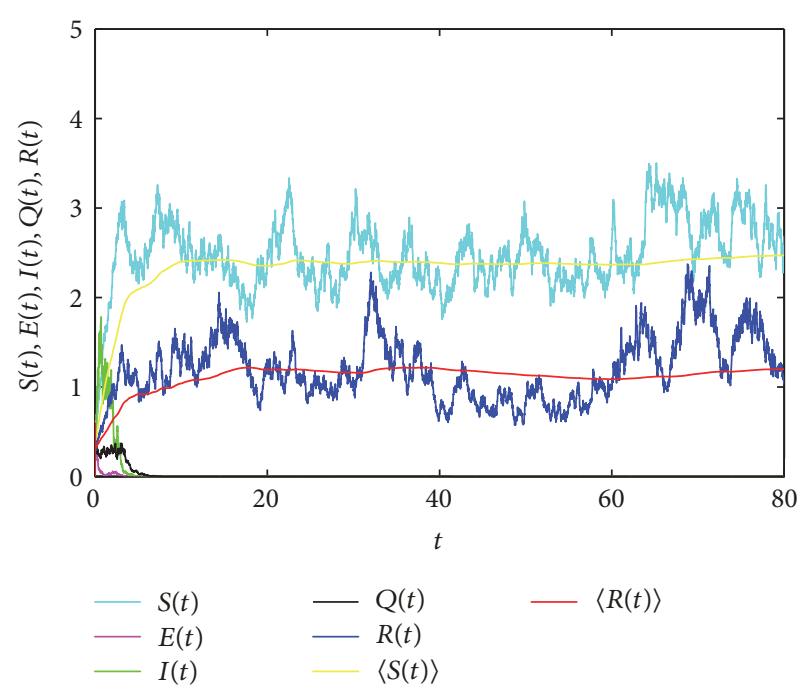

FIGURE 1: Time sequence diagram of system (5) for extinction of the disease.

In Figure 1, take $S(0)=E(0)=I(0)=Q(0)=R(0)=0.3$, $\Lambda=2, \mu=0.55, \beta=0.25, \gamma=0.2, \alpha=2.5, \delta=0.5, p=0.5$, $\varepsilon_{1}=0.1, \varepsilon_{2}=0.1, \lambda=0.18, \varphi=0.15, \sigma_{1}=0.15, \sigma_{2}=1$, $\sigma_{3}=1, \sigma_{4}=0.5, \sigma_{5}=0.25$, and $\Delta t=0.01$. Then

$$
\begin{aligned}
\mu & =0.55>\frac{1}{2}\left(\sigma_{1}^{2} \vee \sigma_{2}^{2} \vee \sigma_{3}^{2} \vee \sigma_{4}^{2} \vee \sigma_{5}^{2}\right)=0.5, \\
\widetilde{R}^{*} & =\frac{2 \alpha(1-\delta p) \beta(\alpha+\mu)}{\left(\lambda+\varepsilon_{1}+\gamma+\mu+\sigma_{3}^{2} / 2\right)(\alpha+\mu)^{2} \wedge\left(\alpha^{2} \sigma_{2}^{2} / 2\right)} \\
& =0.9150<1
\end{aligned}
$$

satisfy the conditions in Theorem 4; we can obtain that the exposed, infected, and quarantined individuals go to extinction almost surely. Moreover,

$$
\begin{aligned}
& \lim _{t \rightarrow \infty}\langle S\rangle=\frac{\Lambda}{\delta p+\mu}=2.5 \\
& \lim _{t \rightarrow \infty}\langle R\rangle=\frac{\delta p \Lambda}{\mu(\delta p+\mu)}=1.1364
\end{aligned}
$$

a.s.

Obviously, Figure 1 supports our results of Theorem 4.

In Figure 2, take $S(0)=E(0)=I(0)=Q(0)=R(0)=0.5$, $\Lambda=0.3, \mu=0.1, \beta=1.5, \gamma=0.2, \alpha=0.3, \delta=0.1, p=0.2$, $\varepsilon_{1}=0.05, \varepsilon_{2}=0.05, \lambda=0.18, \varphi=0.15, \sigma_{1}=\sigma_{2}=\sigma_{3}=\sigma_{4}=$ $\sigma_{5}=0.03$, and $\Delta t=0.01$. Then

$R^{*}$

$$
\begin{aligned}
& =\frac{\mu(1-\delta p) \beta \alpha}{\left(\delta p+\mu+\sigma_{1}^{2} / 2\right)\left(\alpha+\mu+\sigma_{2}^{2} / 2\right)\left(\lambda+\varepsilon_{1}+\gamma+\mu+\sigma_{3}^{2} / 2\right)} \\
& =1.7236>1
\end{aligned}
$$




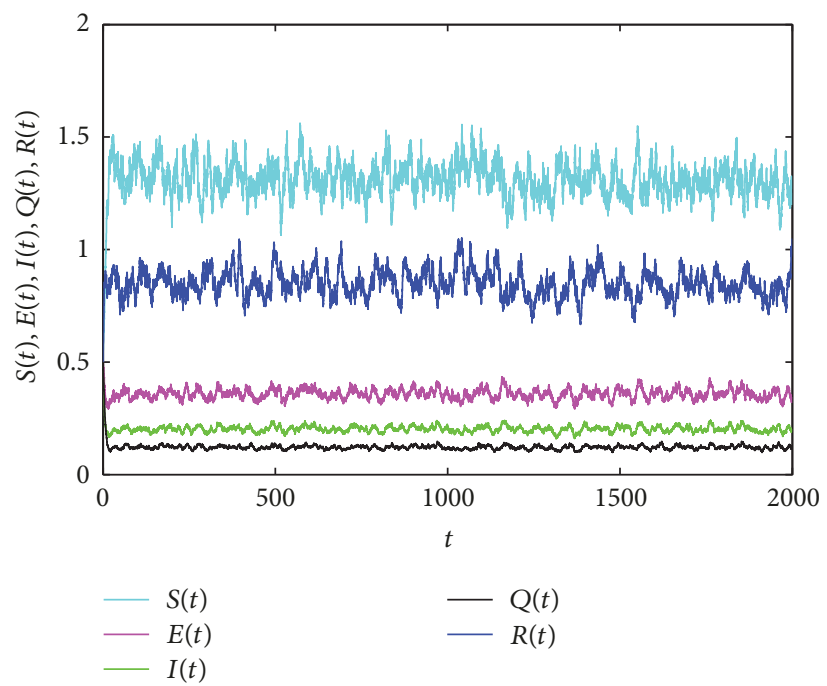

(a)

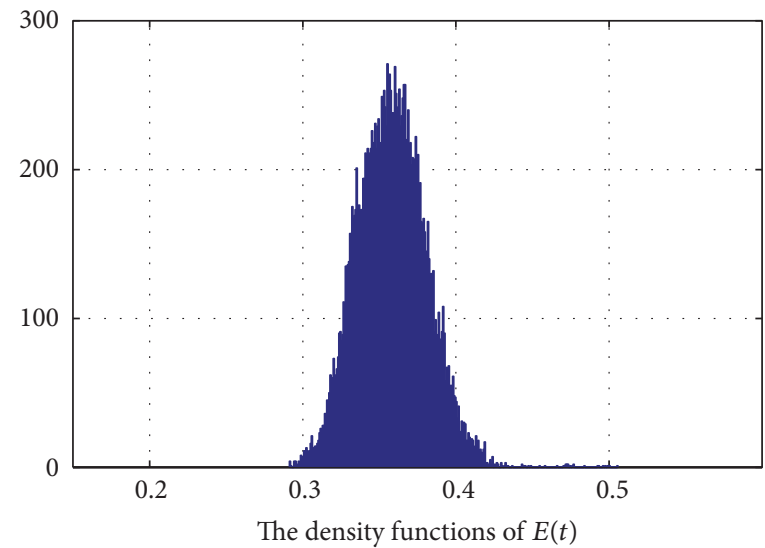

(c)

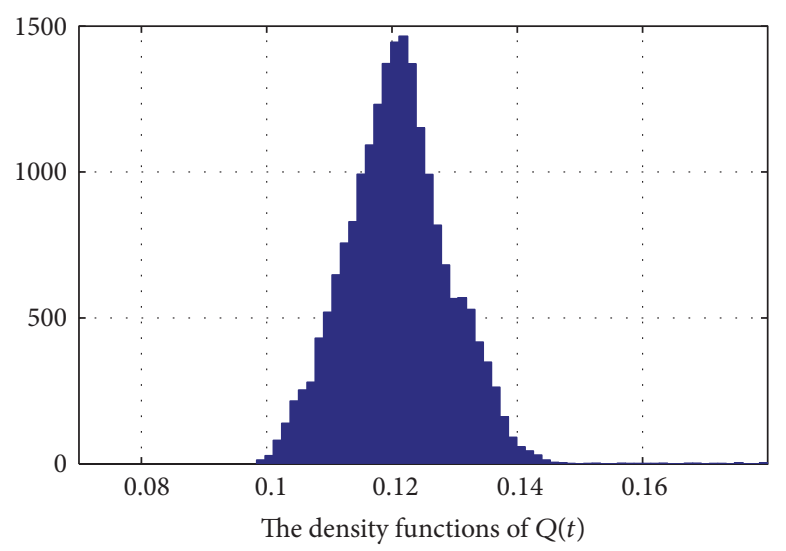

(e)

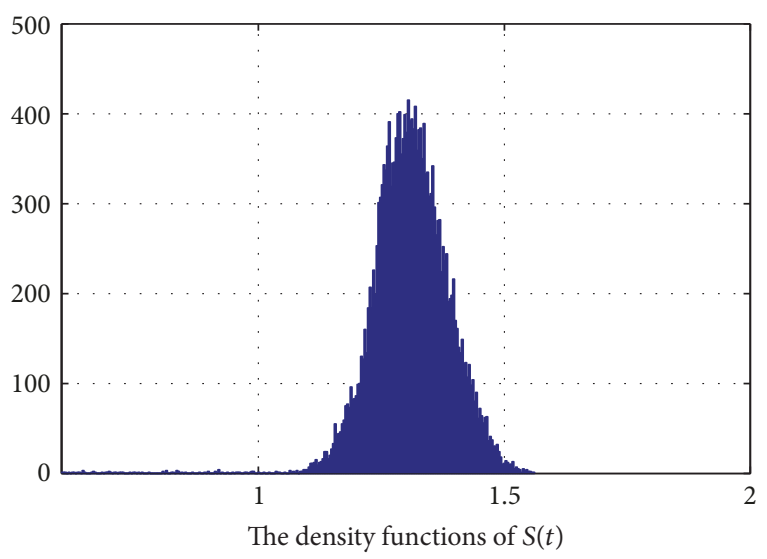

(b)

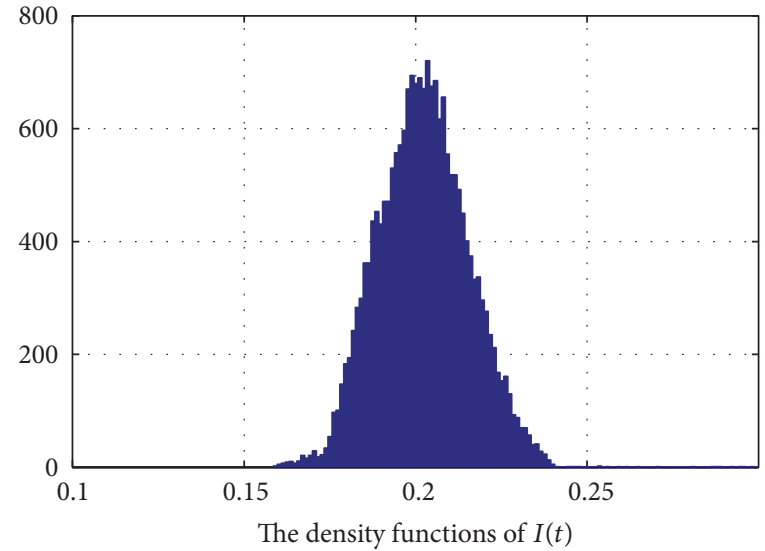

(d)

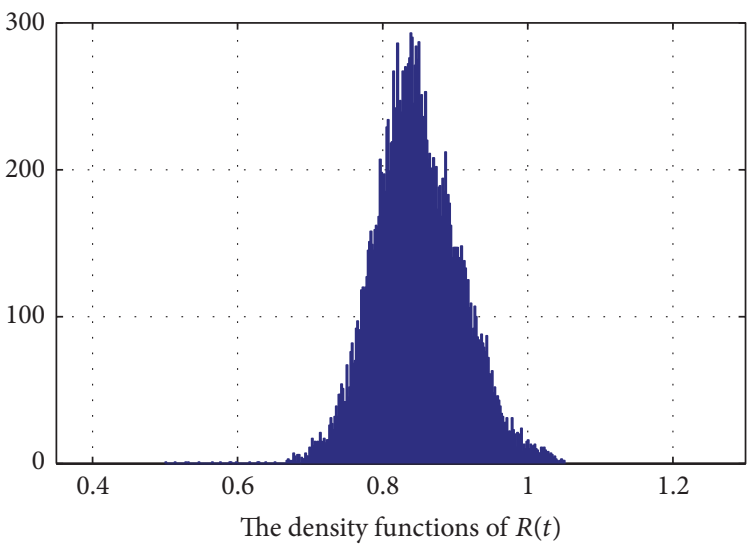

(f)

FIGURE 2: (a) represents the solutions of system (5); (b)-(f) stand for the density functions of $S(t), E(t), I(t), Q(t)$, and $R(t)$, respectively.

satisfies the condition in Theorem 8; we can obtain that system (5) has a unique stationary distribution $\pi(\cdot)$ and it has ergodic property. Figure 2 shows that the solution of system (5) swings up and down in a small neighborhood. According to the density functions in Figures 2(b)-2(f), we can see that there exists a stationary distribution. As expected, Figure 2 confirms our results of Theorem 8 .

In Figure 3, take $S(0)=E(0)=I(0)=Q(0)=R(0)=0.1$, $\Lambda=0.2, \mu=0.55, \beta=2.65, \gamma=0.2, \alpha=2.5, \delta=0.1, p=0.1$, $\varepsilon_{1}=0.1, \varepsilon_{2}=0.1, \lambda=0.18, \varphi=0.15$, and $\Delta t=0.01$. 


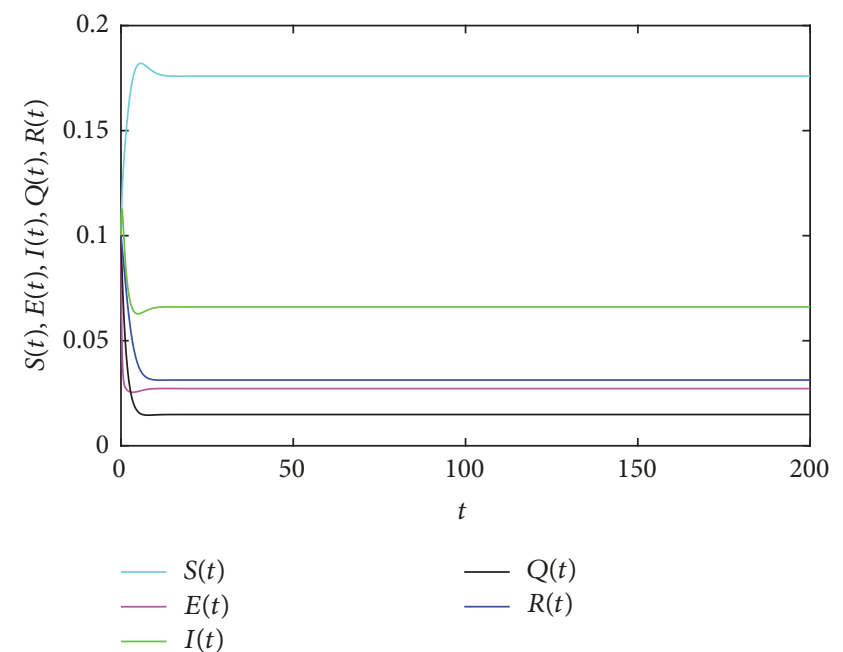

(a)

FIGURE 3: Time sequence diagram of system (5) for persistence and extinction of the disease.

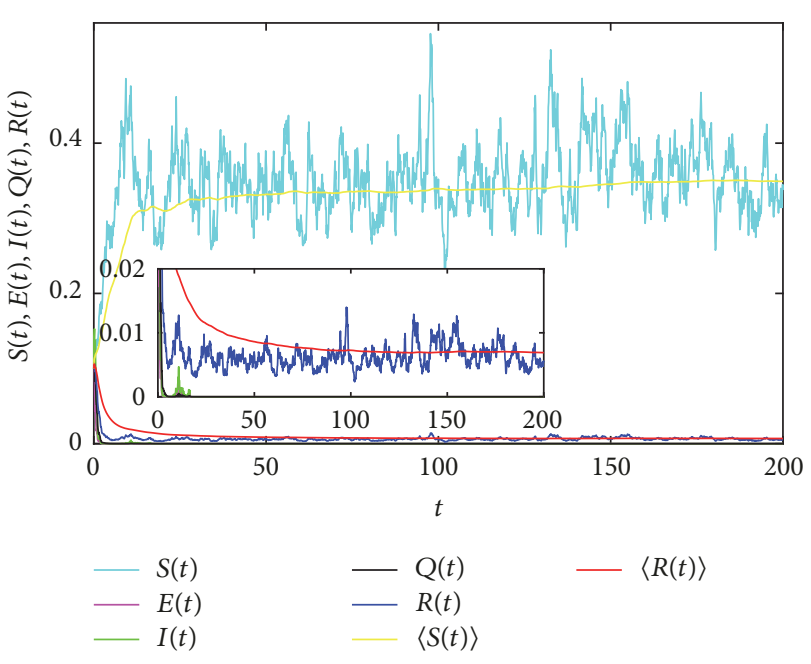

(b)
In Figure 3(a), take $\sigma_{i}=0(i=1,2,3,4,5)$; then

$$
R_{1}=\frac{\mu(1-\delta p) \beta \alpha}{(\delta p+\mu)(\alpha+\mu)\left(\lambda+\varepsilon_{1}+\gamma+\mu\right)}=2.0505>1,
$$

which means the disease will persist. As expected, Figure 3(a) shows the disease persists in real life.

Synchronously, in Figure 3(b), take $\sigma_{1}=0.15, \sigma_{2}=1$, $\sigma_{3}=1, \sigma_{4}=0.5, \sigma_{5}=0.25$. Obviously, Figure 3(b) shows the exposed, infected, and quarantined individuals go to extinction and we can get that the permanent disease of system (3) can die out under stochastic effects. This implies that the stochastic disturbance is conducive to epidemic diseases control.

\section{Conflicts of Interest}

The authors declare that there are no conflicts of interest regarding the publication of this paper.

\section{Acknowledgments}

This work was supported by the National Natural Science Foundation of China (11371230), the SDUST Research Fund (2014TDJH102), and Shandong Provincial Natural Science Foundation, China (ZR2015AQ001).

\section{References}

[1] L. Hörmander, "Hypoelliptic second order differential equations," Acta Mathematica, vol. 119, pp. 147-171, 1967.

[2] J. J. Nieto and D. O’Regan, "Variational approach to impulsive differential equations," Nonlinear Analysis: Real World Applications, vol. 10, no. 2, pp. 680-690, 2009.

[3] Z. Bai and H. Lü, "Positive solutions for boundary value problem of nonlinear fractional differential equation," Journal of Mathematical Analysis and Applications, vol. 311, pp. 495-505, 2005.
[4] J. Mierczynski, "Instability in linear cooperative systems of ordinary differential equations," SIAM Review, vol. 59, pp. 649670, 2017

[5] X. Dong, Z. Bai, and S. Zhang, "Positive solutions to boundary value problems of p-Laplacian with fractional derivative," Boundary Value Problems, vol. 2017, no. 5, 2017.

[6] Y. Cui, "Uniqueness of solution for boundary value problems for fractional differential equations," Applied Mathematics Letters, vol. 51, pp. 48-54, 2016.

[7] Y. Zhang, H. Dong, X. Zhang, and H. Yang, "Rational solutions and lump solutions to the generalized $(3+1)$-dimensional Shallow Water-like equation," Computers \& Mathematics with Applications, vol. 73, pp. 246-252, 2017.

[8] L. Liu and X. Meng, "Optimal harvesting control and dynamics of two-species stochastic model with delays," Advances in Difference Equations, vol. 2017, no. 18, 2017.

[9] Y. Cai, Y. Kang, M. Banerjee, and W. Wang, "A stochastic SIRS epidemic model with infectious force under intervention strategies," Journal of Differential Equations, vol. 259, no. 12, pp. 7463-7502, 2015.

[10] T. Zhang, X. Meng, and T. Zhang, "Global analysis for a delayed SIV model with direct and environmental transmissions," Journal of Applied Analysis and Computation, vol. 6, no. 2, pp. 479491, 2016.

[11] Z. Qiu, "The complete classification for dynamics of an SIS STD model in terms of four parameters," Nonlinear Analysis: Real World Applications, vol. 11, no. 4, pp. 2875-2887, 2010.

[12] W. Ma, M. Song, and Y. Takeuchi, "Global stability of an SIR epidemic model with time delay," Applied Mathematics Letters, vol. 17, no. 10, pp. 1141-1145, 2004.

[13] S. Zhang, X. Meng, T. Feng, and T. Zhang, "Dynamics analysis and numerical simulations of a stochastic non-autonomous predator-prey system with impulsive effects," Nonlinear Analysis: Hybrid Systems, vol. 26, pp. 19-37, 2017.

[14] H. Cheng and T. Zhang, "A new predator-prey model with a profitless delay of digestion and impulsive perturbation on the prey," Applied Mathematics and Computation, vol. 217, no. 22, pp. 9198-9208, 2011. 
[15] L. Liu, X. Meng, and T. Zhang, "Optimal control strategy for an impulsive stochastic competition system with time delays and jumps," Physica A: Statistical Mechanics and its Applications, vol. 477, pp. 99-113, 2017.

[16] Y. He, S. Gao, and D. Xie, "An SIR epidemic model with timevarying pulse control schemes and saturated infectious force," Applied Mathematical Modelling, vol. 37, no. 16-17, pp. 8131-8140, 2013.

[17] T. Zhang, W. Ma, and X. Meng, "Global dynamics of a delayed chemostat model with harvest by impulsive flocculant input," Advances in Difference Equations, vol. 2017, no. 115, 2017.

[18] L. Wu and Z. Feng, "Homoclinic bifurcation in an SIQR model for childhood diseases," Journal of Differential Equations, vol. 168, pp. 150-167, 2000.

[19] S. Li, M. Fan, and X. Rong, "Global threshold dynamics of SIQS epidemic model in time fluctuating environment," International Journal of Biomathematics, vol. 10, no. 4, Article ID 1750060, 22 pages, 2017.

[20] Z. Feng and H. Thieme, "Recurrent outbreaks of childhood diseases revisited: The impact of isolation," Mathematical Biosciences, vol. 123, pp. 93-130, 1995.

[21] H. Hethcote, M. Zhien, and L. Shengbing, "Effects of quarantine in six endemic models for infectious diseases," Mathematical Biosciences, vol. 180, pp. 141-160, 2002.

[22] M. Erdem, M. Safan, and C. Castillo-Chavez, "Mathematical analysis of an SIQR influenza model with imperfect quarantine," Bulletin of Mathematical Biology, vol. 79, no. 7, pp. 1612-1636, 2017.

[23] C. Sun and Y.-H. Hsieh, "Global analysis of an SEIR model with varying population size and vaccination," Applied Mathematical Modelling, vol. 34, no. 10, pp. 2685-2697, 2010.

[24] Z. Qiu and Z. Feng, "Transmission dynamics of an influenza model with vaccination and antiviral treatment," Bulletin of Mathematical Biology, vol. 72, no. 1, pp. 1-33, 2010.

[25] Y. Zhao and D. Jiang, "The threshold of a stochastic SIS epidemic model with vaccination," Applied Mathematics and Computation, vol. 243, pp. 718-727, 2014.

[26] X. Leng, T. Feng, and X. Meng, "Stochastic inequalities and applications to dynamics analysis of a novel SIVS epidemic model with jumps," Journal of Inequalities and Applications, vol. 2017, no. 138, 2017.

[27] S. Gao, L. Chen, J. J. Nieto, and A. Torres, "Analysis of a delayed epidemic model with pulse vaccination and saturation incidence," Vaccine, vol. 24, no. 35-36, pp. 6037-6045, 2006.

[28] X. Meng, L. Chen, and B. Wu, "A delay SIR epidemic model with pulse vaccination and incubation times," Nonlinear Analysis: Real World Applications, vol. 11, no. 1, pp. 88-98, 2010.

[29] J. Jiao, S. Cai, and L. Li, "Impulsive vaccination and dispersal on dynamics of an SIR epidemic model with restricting infected individuals boarding transports," Physica A: Statistical Mechanics and its Applications, vol. 449, pp. 145-159, 2016.

[30] T. Zhang, X. Meng, Y. Song, and T. Zhang, "A stage-structured predator-prey SI model with disease in the prey and impulsive effects," Mathematical Modelling and Analysis, vol. 18, no. 4, pp. 505-528, 2013.

[31] L. Wang and D. Jiang, "A note on the stationary distribution of the stochastic chemostat model with general response functions," Applied Mathematics Letters, vol. 73, pp. 22-28, 2017.

[32] M. Liu and M. Fan, "Permanence of stochastic Lotka-Volterra systems," Journal of Nonlinear Science, vol. 27, no. 2, pp. 425452, 2017.
[33] X. Liu, Y. Li, and W. Zhang, "Stochastic linear quadratic optimal control with constraint for discrete-time systems," Applied Mathematics and Computation, vol. 228, pp. 264-270, 2014.

[34] M. Liu, C. Bai, and Y. Jin, "Population dynamical behavior of a two-predator one-prey stochastic model with time delay," Discrete and Continuous Dynamical Systems - Series A, vol. 37, no. 5, pp. 2513-2538, 2017.

[35] M. Liu and M. Fan, "Stability in distribution of a three-species stochastic cascade predator-prey system with time delays," IMA Journal of Applied Mathematics, vol. 82, no. 2, pp. 396-423, 2017.

[36] H. Ma and Y. Jia, "Stability analysis for stochastic differential equations with infinite Markovian switchings," Journal of Mathematical Analysis and Applications, vol. 435, no. 1, pp. 593-605, 2016.

[37] G. Liu, X. Wang, X. Meng, and S. Gao, "Extinction and persistence in mean of a novel delay impulsive stochastic infected predator-prey system with jumps," Complexity, vol. 2017, Article ID 1950970, pp. 1-15, 2017.

[38] C. Tan and W. Zhang, "On observability and detectability of continuous-time stochastic Markov jump systems," Journal of Systems Science \& Complexity, vol. 28, no. 4, pp. 830-847, 2015.

[39] Y. Zhao and W. Zhang, "Observer-based controller design for singular stochastic Markov jump systems with state dependent noise," Journal of Systems Science \& Complexity, vol. 29, no. 4, pp. 946-958, 2016.

[40] Q. Liu, D. Jiang, N. Shi, T. Hayat, and A. Alsaedi, "Dynamics of a stochastic tuberculosis model with constant recruitment and varying total population size," Physica A: Statistical Mechanics and its Applications, vol. 469, pp. 518-530, 2017.

[41] X. Meng, S. Zhao, T. Feng, and T. Zhang, "Dynamics of a novel nonlinear stochastic SIS epidemic model with double epidemic hypothesis," Journal of Mathematical Analysis and Applications, vol. 433, no. 1, pp. 227-242, 2016.

[42] A. Miao, J. Zhang, T. Zhang, and B. G. S. A. Pradeep, “Threshold Dynamics of a Stochastic SIR Model with Vertical Transmission and Vaccination," Computational and Mathematical Methods in Medicine, vol. 2017, Article ID 4820183, 2017.

[43] L. Wang, Z. Teng, T. Tang, and Z. Li, “Threshold Dynamics in Stochastic SIRS Epidemic Models with Nonlinear Incidence and Vaccination," Computational and Mathematical Methods in Medicine, vol. 2017, Article ID 7294761, 2017.

[44] Y. Chen, B. Wen, and Z. Teng, "The global dynamics for a stochastic SIS epidemic model with isolation," Physica A: Statistical Mechanics and its Applications, vol. 492, pp. 16041624, 2018.

[45] Y. Zhang, K. Fan, S. Gao, and S. Chen, "A remark on stationary distribution of a stochastic SIR epidemic model with double saturated rates," Applied Mathematics Letters, vol. 76, pp. 46-52, 2018.

[46] A. Miao, X. Wang, T. Zhang, W. Wang, and B. Sampath Aruna Pradeep, "Dynamical analysis of a stochastic SIS epidemic model with nonlinear incidence rate and double epidemic hypothesis," Advances in Difference Equations, vol. 2017, no. 226, 2017.

[47] T. Feng, X. Meng, L. Liu, and S. Gao, "Application of inequalities technique to dynamics analysis of a stochastic ecoepidemiology model," Journal of Inequalities and Applications, vol. 2016, no. 327, 2016.

[48] H. Qi, L. Liu, and X. Meng, "Dynamics of a non-autonomous stochastic SIS epidemic model with double epidemic hypothesis," Complexity, vol. 2017, no. 14, 2017. 
[49] F. Wang, B. Chen, C. Lin, and X. Li, "Distributed adaptive neural control for stochastic nonlinear multiagent systems," IEEE Transactions on Cybernetics, vol. 47, no. 7, pp. 1795-1803, 2017.

[50] X. Lv, L. Wang, and X. Meng, "Global analysis of a new nonlinear stochastic differential competition system with impulsive effect," Advances in Difference Equations, vol. 2017, no. 296, 2017.

[51] X. Mao, Stochastic Differential Equations and Applications, Woodhead Publishing, Cambridge, UK, 2007.

[52] Y. Zhao, S. Yuan, and T. Zhang, "The stationary distribution and ergodicity of a stochastic phytoplankton allelopathy model under regime switching," Communications in Nonlinear Science and Numerical Simulation, vol. 37, pp. 131-142, 2016.

[53] J. Yu and M. Liu, "Stationary distribution and ergodicity of a stochastic food-chain model with Lévy jumps," Physica A: Statistical Mechanics and its Applications, vol. 482, pp. 14-28, 2017.

[54] R. Hasminskii, G. Milstein, and M. Nevelon, Stochastic Stability of Differential Equations, Springer-Verlag, Berlin, Germany, 2012.

[55] T. Gard, Introduction to Stochastic Differential Equations, Dekker, New York, NY, USA, 1988.

[56] C. Zhu and G. Yin, "Asymptotic properties of hybrid diffusion systems," SIAM Journal on Control and Optimization, vol. 46, no. 4, pp. 1155-1179, 2007. 


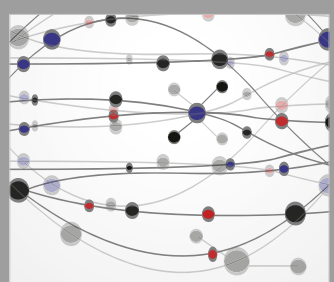

The Scientific World Journal
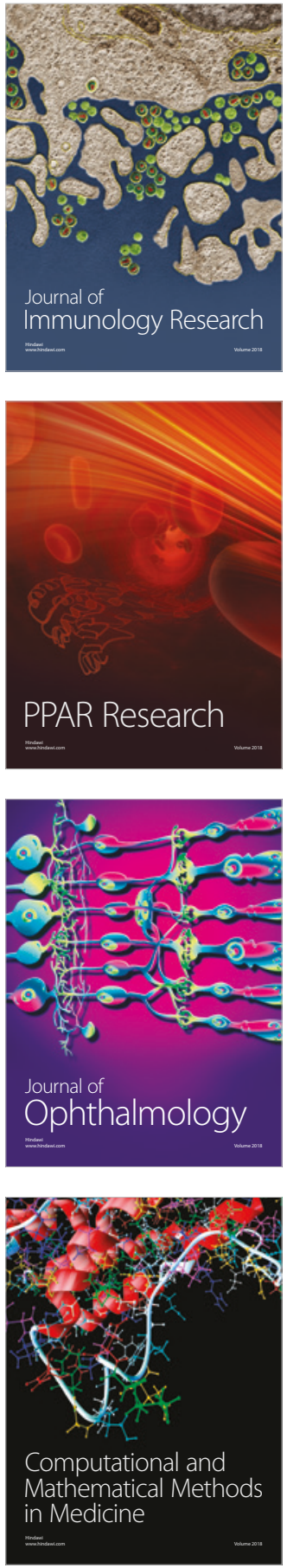

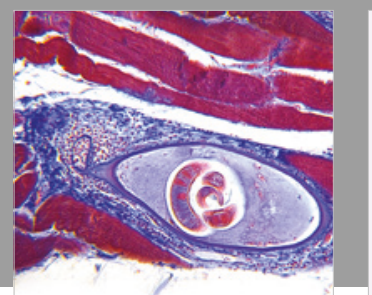

Gastroenterology Research and Practice

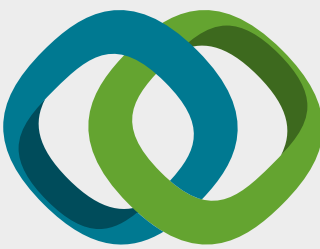

\section{Hindawi}

Submit your manuscripts at

www.hindawi.com
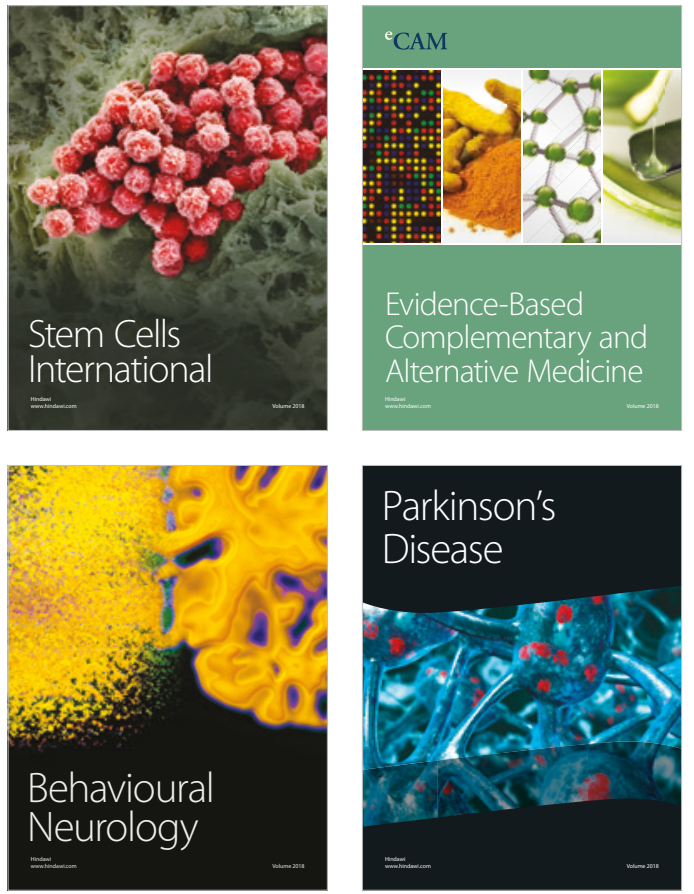

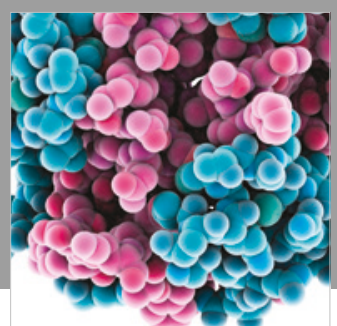

ournal of

Diabetes Research

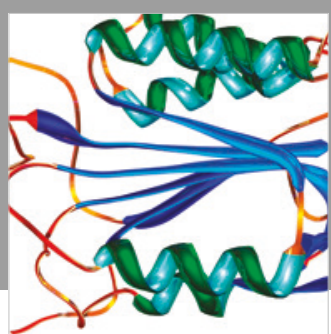

Disease Markers
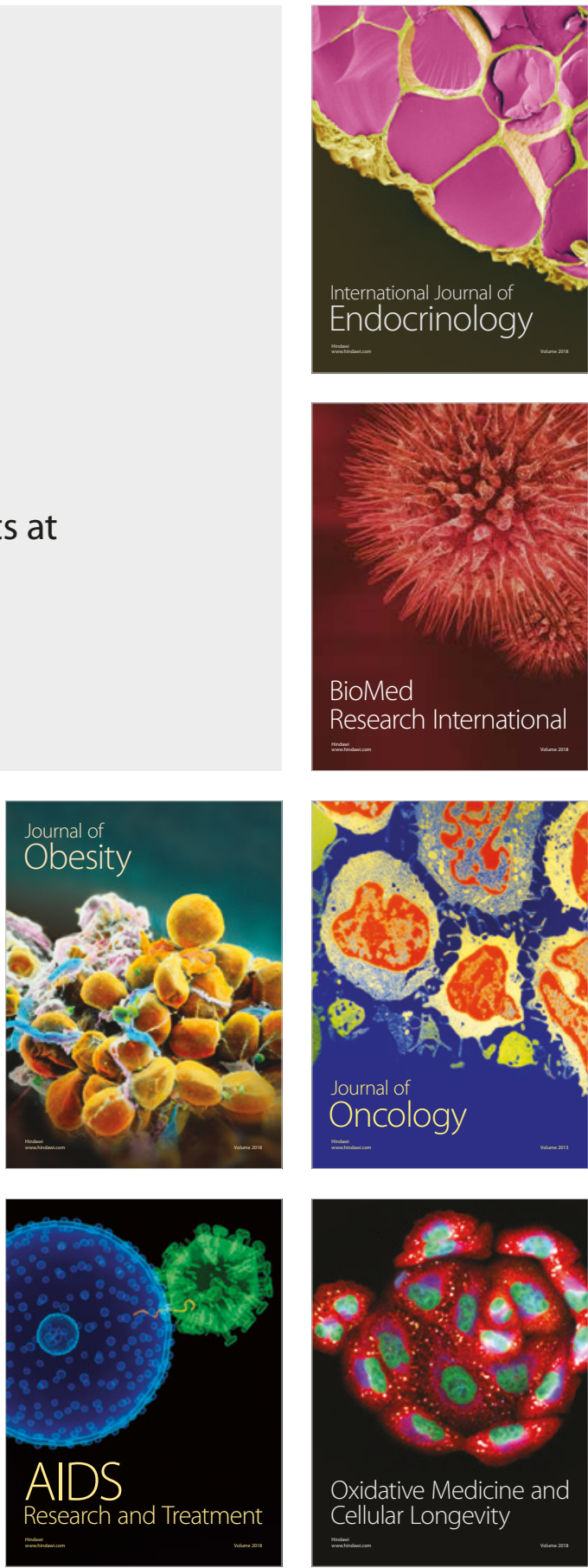